\title{
A NEW CHARACTERIZATION OF GROMOV HYPERBOLICITY FOR NEGATIVELY CURVED SURFACES
}

\author{
José M. RodríGuez ${ }^{(1)}$ AND Eva Tourís(1)(2) \\ Abstract \\ In this paper we show that to check Gromov hyperbolicity of any \\ surface of constant negative curvature, or, Riemann surface, we \\ only need to verify the Rips condition on a very small class of tri- \\ angles, namely, those obtained by marking three points in a simple \\ closed geodesic. This result is, in fact, a new characterization of \\ Gromov hyperbolicity for Riemann surfaces.
}

\section{Introduction}

To understand the connections between graphs and Potential Theory on Riemannian manifolds (see e.g. [ARY], [CFPR], [FR2], [HS], [K1], $[\mathbf{K 2}],[\mathbf{K} 3],[\mathbf{R 1}],[\mathbf{R 2}],[\mathbf{S o}])$ Gromov hyperbolic spaces are a useful tool. Besides, the concept of Gromov hyperbolicity grasps the essence of negatively curved spaces, and has been successfully used in the theory of groups (see e.g. $[\mathbf{G H}],[\mathbf{G 1}],[\mathbf{G} \mathbf{2}]$ and the references therein).

A geodesic metric space is called hyperbolic (in the Gromov sense) if it satisfies the "Rips condition": there is an upper bound of the distance of every point in a side of any geodesic triangle to the union of the two other sides (see Definition 2.3).

But, it is not easy to determine if a given space is Gromov hyperbolic or not. One interesting instance is that of a Riemann surface endowed with the Poincaré metric. With that metric structure a Riemann surface is negatively curved, but not all Riemann surfaces are Gromov hyperbolic, since topological obstacles can impede it: for instance, the two-dimensional jungle-gym (a $\mathbf{Z}^{2}$-covering of a torus with genus two) is not hyperbolic.

2000 Mathematics Subject Classification. 30F, 30F20, 30F45.

Key words. Gromov hyperbolicity, hyperbolic Riemann surface, closed geodesic.

${ }^{(1)}$ Research partially supported by a grant from DGI (BFM 2003-04870), Spain.

${ }^{(2)}$ Research partially supported by a grant from DGI (BFM 2000-0022), Spain. 
We are interested in studying when Riemann surfaces equipped with their Poincaré metric are Gromov hyperbolic. The following theorem is the main result of this paper, which is a new characterization of Gromov hyperbolicity for Riemann surfaces (see Theorem 5.1):

A Riemann surface $S$ is hyperbolic if and only if the $c_{0}$-triangles contained in simple closed geodesics of $S$ satisfy the Rips condition. By a $c_{0}$-triangle we mean a triangle with continuous injective $\left(1, c_{0}\right)$-quasigeodesic sides, and we require that the vertices and the edges of such triangles are contained in simple closed geodesics of $S$.

In general, one has to verify the Rips condition for all triangles. Our result is that for Riemann surfaces you only have to verify it for a smaller class of triangles.

Furthermore this theorem provides a bound for the hyperbolicity constant: if the triangles contained in simple closed geodesics satisfy the Rips condition with constant $\delta_{0}$, then every geodesic triangle satisfy it with constant $\delta=\max \left\{11, \delta_{0}+6\right\}$.

A connected question with our main theorem is when a Euclidean bounded domain with its quasihyperbolic metric is Gromov hyperbolic. (Let us recall that in the case of modulated plane domains, quasihyperbolic and Poincaré metrics are equivalent.) Recently, Balogh and Buckley $[\mathbf{B B}]$ have made significant progress in this question (see also [BHK] and the references therein).

Theorem 5.1 provides good bounds for the hyperbolicity constants of some classical surfaces such as the punctured disk, the annuli, the $Y$-pieces and plane domains of finite type (see Lemma 5.4 and Corollaries 5.1, 5.2 and 5.3).

It can also be successfully used as a powerful tool to study hyperbolicity of a class of Riemann surfaces by means of its decomposition in $Y$-pieces and funnels (see Theorem 5.3).

As a consequence of these results, we have obtained interesting examples of hyperbolic Riemann surfaces (see Theorem 5.3 and Corollaries 5.1, 5.2 and 5.3), and a result that allows us a better understanding of the role that funnels and half-disks (see Definition 5.4) play in the study of hyperbolicity (see Theorem 5.2). Theorem 5.2 is a useful result which has several applications in [RT2] and [PRT2]. One can think of the following as a natural first result in order to study hyperbolicity: if a Riemann surface has a sequence of funnels $\left\{F_{n}\right\}_{n}$ with $\lim _{n \rightarrow \infty} L\left(\partial F_{n}\right)=\infty$, then it is not hyperbolic. In [RT2] we prove that this reasonable result is false indeed, and an important tool in the proof is Theorem 5.2. 
Notations. We denote by $X$ or $X_{n}$ geodesic metric spaces. By $d_{X}$, $L_{X}$ and $B_{X}$ we shall denote, respectively, the distance, the length and the balls in the metric of $X$. From now on, when there is no possible confusion, we will not write the subindex $X$.

We denote by $R, S$ or $S_{0}$ Riemann surfaces. We assume that the metric defined on these surfaces is the Poincaré metric, unless the contrary is specified.

If $\Omega$ is a plane domain, we shall denote by $\lambda_{\Omega}$ the conformal density of the Poincaré metric in $\Omega$, i.e. the function such that $d s=\lambda_{\Omega}(z)|d z|$ is the Poincaré metric in $\Omega$.

We denote by $\Re z$ and $\Im z$ the real and imaginary part of $z$, respectively.

Finally, we denote by $l, c$ and $c_{i}$, positive constants which can assume different values in different theorems.

Acknowledgements. We would like to thank Professor J. L. Fernández for some useful discussions. Also, we would like to thank the referee for his/her careful reading of the manuscript and for some helpful suggestions.

\section{Background in Gromov spaces}

In our study of hyperbolic Gromov spaces we use the notations of $[\mathbf{G H}]$. We give now the basic facts about these spaces. We refer to $[\mathbf{G H}]$ for more background and further results.

Definition 2.1. Let us fix a point $w$ in a metric space $(X, d)$. We define the Gromov product of $x, y \in X$ with respect to the point $w$ as

$$
(x \mid y)_{w}:=\frac{1}{2}(d(x, w)+d(y, w)-d(x, y)) \geq 0 .
$$

We say that the metric space $(X, d)$ is $\delta$-hyperbolic $(\delta \geq 0)$ if

$$
(x \mid z)_{w} \geq \min \left\{(x \mid y)_{w},(y \mid z)_{w}\right\}-\delta,
$$

for every $x, y, z, w \in X$. We say that $X$ is hyperbolic (in the Gromov sense) if the value of $\delta$ is not important.

It is convenient to remark that this definition of hyperbolicity is not universally accepted, since sometimes the word hyperbolic refers to negative curvature or to the existence of Green's function. However, in this paper we only use the word hyperbolic in the sense of Definition 2.1.

Examples. (1) Every bounded metric space $X$ is (diam $X)$-hyperbolic. 
(2) Every complete simply connected Riemannian manifold with sectional curvature which is bounded from above by $-k$, with $k>0$, is hyperbolic.

(3) Every tree with edges of arbitrary length is 0-hyperbolic.

We refer the reader to $[\mathbf{B H K}],[\mathbf{G H}]$ and $[\mathbf{C D P}]$ for further examples.

Definition 2.2. If $\gamma:[a, b] \longrightarrow X$ is a continuous curve in a metric space $(X, d)$, we can define the length of $\gamma$ as

$$
L(\gamma):=\sup \left\{\sum_{i=1}^{n} d\left(\gamma\left(t_{i-1}\right), \gamma\left(t_{i}\right)\right): a=t_{0}<t_{1}<\cdots<t_{n}=b\right\} .
$$

We say that $\gamma$ is a geodesic if it is an isometry, i.e.

$L\left(\left.\gamma\right|_{[t, s]}\right)=d(\gamma(t), \gamma(s))=|t-s|$ for every $s, t \in[a, b]$. We say that $\gamma$ is a local geodesic if for every $t \in[a, b]$ there exists $\varepsilon>0$ such that the restriction of $\gamma$ to $[t-\varepsilon, t+\varepsilon] \cap[a, b]$ is a geodesic. We say that $X$ is a geodesic metric space if for every $x, y \in X$ there exists a geodesic joining $x$ and $y$; we denote by $[x, y]$ any such geodesic (since we do not require uniqueness of geodesics, this notation is ambiguous, but it is convenient).

Definition 2.3. If $X$ is a geodesic metric space and $J$ is a polygon whose sides are $J_{1}, J_{2}, \ldots, J_{n}$, with $J_{j} \subseteq X$, we say that $J$ is $\delta$-thin if for every $x \in J_{i}$ we have that $d\left(x, \cup_{j \neq i} J_{j}\right) \leq \delta$. If $x_{1}, x_{2}, x_{3} \in X$, a geodesic triangle $T=\left\{x_{1}, x_{2}, x_{3}\right\}$ is the union of three geodesics $J_{1}:=\left[x_{1}, x_{2}\right]$, $J_{2}:=\left[x_{2}, x_{3}\right]$ and $J_{3}:=\left[x_{3}, x_{1}\right]$. The space $X$ is $\delta$-thin (or satisfies the Rips condition with constant $\delta$ ) if every geodesic triangle in $X$ is $\delta$-thin.

Remark. Every geodesic quadrilateral in a $\delta$-thin geodesic space is $2 \delta$-thin. To see this, it is enough to divide the quadrilateral in two triangles. In general, every geodesic polygon of $n$ sides is $(n-2) \delta$-thin. If we have a triangle with two identical vertices, we call it a "bigon"; obviously, every bigon in a $\delta$-thin space is $\delta$-thin.

A fundamental result is that hyperbolicity is equivalent to the Rips condition:

Theorem A ([GH, p. 41]). Let us consider a geodesic metric space $X$.

(1) If $X$ is $\delta$-hyperbolic, then it is $4 \delta$-thin.

(2) If $X$ is $\delta$-thin, then it is $4 \delta$-hyperbolic.

We present now the class of maps which play the main role in the theory. 
Definition 2.4. A function between two metric spaces $f: X \longrightarrow Y$ is a quasi-isometry if there are constants $a \geq 1, b \geq 0$ with

$$
\frac{1}{a} d_{X}\left(x_{1}, x_{2}\right)-b \leq d_{Y}\left(f\left(x_{1}\right), f\left(x_{2}\right)\right) \leq a d_{X}\left(x_{1}, x_{2}\right)+b,
$$

for every $x_{1}, x_{2} \in X$.

Such a function is called an $(a, b)$-quasi-isometry. An $(a, b)$-quasigeodesic in $X$ is an $(a, b)$-quasi-isometry between an interval of $\mathbf{R}$ and $X$.

Let us observe that a quasi-isometry does not have to be continuous (for instance, the map $f: \mathbf{R} \longrightarrow \mathbf{Z}$ such that $f([n, n+1)$ ) $=n$ for every integer $n$ is a $(1,1)$-quasi-isometry).

Quasi-isometries are important since they are maps which preserve hyperbolicity:

Theorem B ([GH, p. 88]). Let us consider an $(a, b)$-quasi-isometry between two geodesic metric spaces $f: X \longrightarrow Y$. If $Y$ is $\delta$-hyperbolic, then $X$ is $\delta^{\prime}$-hyperbolic, where $\delta^{\prime}$ is a constant which depends only on $\delta$, $a$ and $b$.

Definition 2.5. Let us consider $H>0$, a metric space $X$, and subsets $Y, Z \subseteq X$. The set $V_{H}(Y):=\{x \in X: d(x, Y) \leq H\}$ is called the $H$-neighbourhood of $Y$ in $X$. The Hausdorff distance of $Y$ to $Z$ is defined by $\mathcal{H}(Y, Z):=\inf \left\{H>0: Y \subseteq V_{H}(Z), Z \subseteq V_{H}(Y)\right\}$.

The following is a beautiful and useful result:

Theorem C ([GH, p. 87]). For each $\delta, b \geq 0$ and $a \geq 1$, there exists $a$ constant $H=H(\delta, a, b)$ with the following property:

Let us consider a $\delta$-hyperbolic geodesic metric space $X$ and an $(a, b)$ quasigeodesic $g$ joining $x$ and $y$. If $\gamma$ is a geodesic joining $x$ and $y$, then $\mathcal{H}(g, \gamma) \leq H$.

This property is known as geodesic stability. Mario Bonk has proved that, in fact, geodesic stability is equivalent to hyperbolicity [Bo].

Along this paper we will work with topological subspaces of a geodesic metric space $X$. There is a natural way to define a distance in these spaces:

Definition 2.6. If $X_{0}$ is a subset connected by rectifiable paths of a metric space $(X, d)$, then we associate to it the inner or intrinsic distance

$$
\begin{aligned}
d_{X_{0}}(x, y) & :=\left.d_{X}\right|_{X_{0}}(x, y) \\
& :=\inf \left\{L(\gamma): \gamma \subset X_{0} \text { is a continuous curve joining } x \text { and } y\right\} \\
& \geq d_{X}(x, y) .
\end{aligned}
$$




\section{Results in metric spaces}

We are interested in studying when non-exceptional Riemann surfaces equipped with their Poincaré metric are Gromov hyperbolic. However, we have proved several results on hyperbolicity for general metric spaces, which are interesting by themselves and have consequences for Riemann surfaces (see Section 5).

We want to remark that almost every constant appearing in the results of this paper depends just on a small number of parameters (in fact, we give explicit expressions for them). This is a common place in the theory of hyperbolic spaces (see e.g. Theorems A, B and C) and is also typical of surfaces with curvature -1 (see the Collar Lemma in $[\mathbf{R}]$ and $[\mathbf{S}]$, and Theorem 3.1 in [PRT2]).

We need some technical results which we collect in the following lemmas.

Lemma 3.1. Let us consider a geodesic metric space $X$ and $\varepsilon>0$. If $\gamma$ is a continuous curve joining $x, y \in X$ with $L_{X}(\gamma) \leq d_{X}(x, y)+\varepsilon$, then $\gamma$ is a $(1, \varepsilon)$-quasigeodesic with its arc-length parametrization.

Proof: Let us consider $\gamma$ with its arc-length parametrization $\gamma:[0, l] \longrightarrow$ $X$. Since $\gamma$ is continuous, it is clear that $d_{X}(\gamma(t), \gamma(s)) \leq L_{X}(\gamma([t, s]))=$ $|t-s|$. Let us show now $|t-s| \leq d_{X}(\gamma(t), \gamma(s))+\varepsilon$. We assume that there are $0 \leq t, s \leq l$ with $|t-s|>d_{X}(\gamma(t), \gamma(s))+\varepsilon$. Without loss of generality we can assume $t<s$. We define a curve $\gamma_{0}$ as a concatenation of three curves: $\gamma([0, t])$, a geodesic $\eta$ connecting $\gamma(t)$ with $\gamma(s)$, and $\gamma([s, l])$. Since $\gamma_{0}$ is a continuous curve connecting $x$ with $y$, we have that

$$
\begin{aligned}
d_{X}(x, y) & \leq L_{X}\left(\gamma_{0}\right)=L_{X}(\gamma)-L_{X}(\gamma([t, s]))+d_{X}(\gamma(t), \gamma(s)) \\
& =L_{X}(\gamma)-|t-s|+d_{X}(\gamma(t), \gamma(s)) \\
& <L_{X}(\gamma)-\varepsilon \leq d_{X}(x, y)
\end{aligned}
$$

which is a contradiction.

Corollary 3.1. Let us consider a geodesic metric space $X$ and $\varepsilon>0$. If $\gamma$ is a continuous curve with $L_{X}(\gamma) \leq \varepsilon$, then $\gamma$ is a $(1, \varepsilon)$-quasigeodesic with its arc-length parametrization.

Lemma 3.2. Let us consider a metric space $X$ with a closed geodesic $g$ of length $l$. If $\gamma$ is a continuous injective $(1, c)$-quasigeodesic in $X$ with its arc-length parametrization, and it is contained in $g$, then $L(\gamma) \leq$ $(l+c) / 2$. 
Remarks. 1. It is clear that every closed geodesic is only a local geodesic, but not a geodesic (see Definition 2.2); however, since there is no possible confusion, we call it closed geodesic instead of closed local geodesic.

2. If $\gamma$ is a geodesic, it is clear that $L(\gamma) \leq l / 2$; Lemma 3.2 generalizes this fact.

Proof: Let us consider $\gamma$ with its arc-length parametrization $\gamma:\left[0, l_{0}\right] \longrightarrow$ $X$. Assume that $l_{0}>(l+c) / 2$; then $l-l_{0}<l_{0}-c$. Observe that $d\left(\gamma(0), \gamma\left(l_{0}\right)\right) \leq l-l_{0}$, since $g \backslash \gamma$ is a continuous curve of length $l-l_{0}$ joining $\gamma(0)$ and $\gamma\left(l_{0}\right)$ ( $\gamma$ is continuous and injective). Hence, we have that $l_{0}-c \leq d\left(\gamma(0), \gamma\left(l_{0}\right)\right) \leq l-l_{0}<l_{0}-c$, which is a contradiction.

Lemma 3.3. Every $(a, b)$-quasigeodesic triangle in a $\delta$-hyperbolic geodesic metric space $X$ is $(4 \delta+2 H(\delta, a, b))$-thin, where $H$ is the constant in Theorem $C$.

Proof: Given an $(a, b)$-quasigeodesic triangle in $X$ of sides $q_{1}, q_{2}, q_{3}$, Theorem $\mathrm{C}$ gives that there exist geodesics $g_{1}, g_{2}, g_{3}$, such that $g_{i}$ has the same end points as $q_{i}$ and $\mathcal{H}\left(g_{i}, q_{i}\right) \leq H=H(\delta, a, b)$. If $\{i, j, k\}$ is any permutation of $\{1,2,3\}$, and $x \in q_{i}$, then there is a point $x^{\prime} \in g_{i}$ with $d\left(x, x^{\prime}\right) \leq H$. Since $X$ is $4 \delta$-thin, we can find $y^{\prime} \in g_{j} \cup g_{k}$ with $d\left(y^{\prime}, x^{\prime}\right) \leq 4 \delta$. We also have a point $y \in q_{j} \cup q_{k}$ with $d\left(y^{\prime}, y\right) \leq H$. Consequently $d\left(x, q_{j} \cup q_{k}\right) \leq d(x, y) \leq 4 \delta+2 H$.

The following result is a modification of Theorem 2.4 in [RT1] (using a completely different line of argument). Furthermore, this proof gives an explicit expression for the constants involved. It can be applied to the study of hyperbolicity of Riemann surfaces (see Theorem 5.3). In order to state it, we need one definition.

Definition 3.1. We say that the closed geodesic metric spaces $\left\{X_{n}\right\}_{n \in \Lambda}$ are a $\left(c_{1}, c_{2}\right)$-regular decomposition of the geodesic metric space $X$ if they verify the following conditions:

(a) $X=\cup_{n \in \Lambda} X_{n}$ and $X_{n} \cap X_{m}=\eta_{n m}$, where for each $n \in \Lambda$, $\left\{\eta_{n m}\right\}_{m \in \Lambda \backslash\{n\}}$ are pairwise disjoint closed subsets of $X_{n}\left(\eta_{n m}=\varnothing\right.$ is allowed); furthermore any geodesic in $X$ with finite length meets at most a finite number of $\eta_{n m}$ 's.

(b) For any $n, m \in \Lambda$, $\operatorname{diam}_{X_{n}}\left(\eta_{n m}\right) \leq c_{1}$ and if $\eta_{n m} \neq \varnothing$, then $X \backslash \eta_{n m}$ is not connected and $a, b$ are in different connected components of $X \backslash \eta_{n m}$ for any $a \in X_{n} \backslash \eta_{n m}, b \in X_{m} \backslash \eta_{n m}$. 
(c) For each $n \in \Lambda$ there exist disjoint sets $A_{n}, B_{n} \subseteq \Lambda$, verifying the following properties: if $m \notin A_{n} \cup B_{n}$, then $\eta_{n m}=\varnothing$; $\operatorname{diam}_{X_{n}}\left(\cup_{m \in A_{n}} \eta_{n m}\right) \leq c_{2}$; and every geodesic joining two points in $X_{n}$ cannot escape from $X_{n}$ across a $\eta_{n m}$ with $m \in B_{n}$.

Remarks. $\quad 1$. The sets $\Lambda, A_{n}$ and $B_{n}$ do not need to be countable.

2. The hypothesis on $X \backslash \eta_{n m}$ guarantees that the graph $R=(V, E)$ constructed in the following way is a tree: $V=\cup_{n \in \Lambda}\left\{v_{n}\right\}$ and $\left[v_{n}, v_{m}\right] \in E$ if and only if $\eta_{n m} \neq \varnothing$.

3. We can think that the hypothesis "a geodesic joining two points in $X_{n}$ cannot escape from $X_{n}$ across a $\eta_{n m}$ with $m \in B_{n}$ ", is very restrictive; however, Lemma 5.5 below gives a very simple condition which allows one to assure this hypothesis.

4. If $X$ is a Riemann surface, $\left\{X_{n}\right\}_{n \in \Lambda}$ are bordered Riemann surfaces and $\eta_{n m} \subset \partial X_{n} \cap \partial X_{m}$, condition " $a, b$ are in different components of $X \backslash \eta_{n m}$ for any $a \in X_{n} \backslash \eta_{n m}, b \in X_{m} \backslash \eta_{n m}$ " in (b), is a consequence of " $X \backslash \eta_{n m}$ is not connected".

5. We wish to emphasize that condition $\operatorname{diam}_{X_{n}}\left(\eta_{n m}\right) \leq c_{1}$ is not very restrictive: if the space is "wide" at every point (in the sense of long injectivity radius, as in the case of simply connected spaces) or "narrow" at every point (as in the case of trees), it is easier to study its hyperbolicity; if we can found narrow parts (as $\eta_{n m}$ ) and wide parts, the problem is more difficult and interesting.

Theorem 3.1. Let us consider a $\left(c_{1}, c_{2}\right)$-regular decomposition $\left\{X_{n}\right\}_{n \in \Lambda}$ of the geodesic metric space $X$. If there exists a constant $\delta_{0}$ such that $X_{n}$ is $\delta_{0}$-thin for every $n \in \Lambda$, then $X$ is $\delta$-thin with $\delta=20 \delta_{0}+\max \left\{c_{1}+\right.$ $\left.c_{2} / 2, c_{2}\right\}$.

Proof: Let us consider a geodesic triangle $T=\{a, b, c\}$ in $X$. If $T \subseteq X_{n}$ for some $n$, then $T$ is $\delta_{0}$-thin, by hypothesis. We assume now that $T$ intersects several $X_{n}$ 's. We intend to study $T$ in each of those $X_{n}$ 's separately.

Let us take $z \in T$. If $z$ belongs to two sides of $T$, there is nothing to prove; if $z$ only belongs to one side of $T$, we denote by $A$ the union of the sides of $T$ which do not intersect $z$.

Let us fix $n \in \Lambda$. We assume first that the three sides of $T$ intersect $X_{n}$

We construct a geodesic polygon $P_{n}$ in $X_{n}$ modifying $T \cap X_{n}$ in the following way: Let us consider a side $\gamma_{i}(i=1,2,3)$ of $T$. If $\gamma_{i} \subseteq X_{n}$, we define $g_{i}:=\gamma_{i}$. If $\gamma_{i}$ is not contained in $X_{n}$, then we consider 
$\gamma_{i}:[0, l] \longrightarrow X$. Let us define

$$
\begin{aligned}
& t_{1}^{i}:=\min \left\{0 \leq t \leq l: \gamma_{i}(t) \in X_{n}\right\}, \\
& t_{4}^{i}:=\max \left\{0 \leq t \leq l: \gamma_{i}(t) \in X_{n}\right\} .
\end{aligned}
$$

If $\gamma_{i}\left(\left[t_{1}^{i}, t_{4}^{i}\right]\right) \subseteq X_{n}$, we consider $g_{i}:=\gamma_{i}\left(\left[t_{1}^{i}, t_{4}^{i}\right]\right)$. In other case, we define

$$
\begin{aligned}
& t_{2}^{i}:=\min \left\{0 \leq t \leq l: \gamma_{i}(t) \in \cup_{m \in A_{n}} \eta_{n m}\right\}, \\
& t_{3}^{i}:=\max \left\{0 \leq t \leq l: \gamma_{i}(t) \in \cup_{m \in A_{n}} \eta_{n m}\right\},
\end{aligned}
$$

and $g_{i}:=\gamma_{i}\left(\left[t_{1}^{i}, t_{2}^{i}\right]\right) \cup\left[\gamma_{i}\left(t_{2}^{i}\right), \gamma_{i}\left(t_{3}^{i}\right)\right] \cup \gamma_{i}\left(\left[t_{3}^{i}, t_{4}^{i}\right]\right)$, where we choose a geodesic $\left[\gamma_{i}\left(t_{2}^{i}\right), \gamma_{i}\left(t_{3}^{i}\right)\right]$ in $X_{n}$. This minimum and this maximum exist since $\gamma_{i}$ is a continuous function in a compact interval and $\gamma_{i} \cap\left(\cup_{m \in A_{n}} \eta_{n m}\right)$ is a compact set: each $\eta_{n m}$ is a closed set and $\gamma_{i}$ meets at most a finite number of $\eta_{n m}$ 's.

It is possible that $g_{1} \cup g_{2} \cup g_{3}$ is not a polygon, since there can exist gaps between two $g_{i}$ 's. Since $\operatorname{diam}_{X_{n}}\left(\eta_{n m}\right) \leq c_{1}$ and $X \backslash \eta_{n m}$ is not connected for any $m \in \Lambda$, we can find three geodesics $h_{1}, h_{2}, h_{3}$ in $X_{n}$ of length less or equal than $c_{1}$ such that $g_{1} \cup h_{1} \cup g_{2} \cup h_{2} \cup g_{3} \cup h_{3}$ is a geodesic polygon $P_{n}$ in $X_{n}$ (some $h_{i}$ can be a point). It is clear that $P_{n}$ has at most 12 sides, and then it is $10 \delta_{0}$-thin.

Without loss of generality we can assume that $z \in g_{1}$. In order to simplify the notation, we define $x_{j}:=\gamma_{1}\left(t_{j}^{1}\right)$ for $1 \leq j \leq 4$.

If $g_{1}:=\gamma_{1}\left(\left[t_{1}^{1}, t_{4}^{1}\right]\right)=\left[x_{1}, x_{4}\right]$, then there exists $w^{\prime} \in P_{n} \backslash \operatorname{int} g_{1}$ with $d_{X_{n}}\left(z, w^{\prime}\right) \leq 10 \delta_{0}$, where int $g_{1}$ denotes $g_{1}$ without its end points. If $w^{\prime} \in A$, then $d_{X}(z, A) \leq 10 \delta_{0}$; if $w^{\prime} \notin A$, then there exists $w \in$ $P_{n} \cap A$ with $d_{X_{n}}\left(w, w^{\prime}\right) \leq \max \left\{c_{1}, c_{2} / 2\right\}$, and therefore $d_{X}(z, A) \leq$ $10 \delta_{0}+\max \left\{c_{1}, c_{2} / 2\right\}$.

Let us assume now that $g_{1}:=\left[x_{1}, x_{2}\right] \cup\left[x_{2}, x_{3}\right] \cup\left[x_{3}, x_{4}\right]$. Recall that $\left[x_{1}, x_{2}\right] \cup\left[x_{3}, x_{4}\right] \subseteq \gamma_{i} \subseteq T$, and $L_{X}\left(\left[x_{2}, x_{3}\right]\right) \leq c_{2}$. We denote by $a_{1} \in\left[x_{1}, x_{2}\right]$ the point farther of $x_{2}$ such that $d_{X_{n}}\left(a_{1},\left[x_{2}, x_{3}\right]\right) \leq 10 \delta_{0}$; in a similar way, we define $a_{2} \in\left[x_{3}, x_{4}\right]$ as the point farther of $x_{3}$ such that $d_{X_{n}}\left(a_{2},\left[x_{2}, x_{3}\right]\right) \leq 10 \delta_{0}$; then $d_{X_{n}}\left(a_{1}, a_{2}\right) \leq 20 \delta_{0}+c_{2}$.

Let us consider $b_{1} \in\left[a_{1}, x_{1}\right]$ the point farther of $a_{1}$ such that $d_{X_{n}}\left(b_{1},\left[x_{3}, x_{4}\right]\right) \leq 10 \delta_{0}$ (if this $b_{1}$ does not exist, we take $b_{1}:=a_{1}$ ) and $b_{2} \in\left[a_{2}, x_{4}\right]$ the point farther of $a_{2}$ such that $d_{X_{n}}\left(b_{2},\left[x_{1}, x_{2}\right]\right) \leq 10 \delta_{0}$ (if this $b_{2}$ does not exist, we take $\left.b_{2}:=a_{2}\right)$. If $b_{1} \neq a_{1}$, then $d_{X}\left(b_{1}, x_{3}\right)=$ $L_{X}\left(\left[b_{1}, x_{3}\right]\right)=d_{X}\left(b_{1},\left[x_{3}, x_{4}\right]\right) \leq 10 \delta_{0}$; in a similar way, if $b_{2} \neq a_{2}$, then $d_{X}\left(b_{2}, x_{2}\right) \leq 10 \delta_{0}$. We consider now the next four possibilities: 
- If $b_{1}=a_{1}$ and $b_{2}=a_{2}$, we have seen that $d_{X}\left(b_{1}, b_{2}\right) \leq 20 \delta_{0}+c_{2}$.

- If $b_{1} \neq a_{1}$ and $b_{2} \neq a_{2}$, then $d_{X}\left(b_{1}, b_{2}\right) \leq L_{X}\left(\left[b_{1}, x_{3}\right]\right)+L_{X}\left(\left[b_{2}, x_{2}\right]\right) \leq$ $20 \delta_{0}$.

- If $b_{1} \neq a_{1}$ and $b_{2}=a_{2}$, then there is a point $z_{0} \in\left[x_{2}, x_{3}\right]$ with $d_{X}\left(b_{2}, z_{0}\right) \leq 10 \delta_{0}$; since there is some $x_{j}(j=2,3)$ with $d_{X}\left(x_{j}, z_{0}\right) \leq$ $c_{2} / 2$, we obtain that $d_{X}\left(b_{1}, b_{2}\right) \leq d_{X}\left(b_{1}, x_{j}\right)+d_{X}\left(x_{j}, z_{0}\right)+d_{X}\left(z_{0}, b_{2}\right) \leq$ $20 \delta_{0}+c_{2} / 2$.

- If $b_{1}=a_{1}$ and $b_{2} \neq a_{2}$, we obtain in a similar way that $d_{X}\left(b_{1}, b_{2}\right) \leq$ $20 \delta_{0}+c_{2} / 2$.

Therefore, in the four situations we have $d_{X}\left(b_{1}, b_{2}\right) \leq 20 \delta_{0}+c_{2}$. If $z \in\left[b_{1}, x_{1}\right] \cup\left[b_{2}, x_{4}\right]$, then $d_{X}(z, A) \leq 10 \delta_{0}+\max \left\{c_{1}, c_{2} / 2\right\}$. If $z \in\left[b_{1}, b_{2}\right]$, we can take $b_{i}$ with $d_{X}\left(z, b_{i}\right) \leq 10 \delta_{0}+c_{2} / 2$; since $d_{X}\left(b_{i}, A\right) \leq 10 \delta_{0}+$ $\max \left\{c_{1}, c_{2} / 2\right\}$, we obtain $d_{X}(z, A) \leq 20 \delta_{0}+\max \left\{c_{1}+c_{2} / 2, c_{2}\right\}$. Let us remark that if we consider $z^{\prime} \in\left[b_{1}, b_{2}\right]$, with $z^{\prime} \notin X_{n}$, the same argument gives $d_{X}\left(z^{\prime}, A\right) \leq 20 \delta_{0}+\max \left\{c_{1}+c_{2} / 2, c_{2}\right\}$.

Let us assume now that only two sides of $T$ intersect $X_{n}$. As in the previous case, we can replace each $\gamma_{i}$ which intersect $X_{n}$ by $g_{i}$. Then we can construct in a similar way a geodesic polygon $P_{n}$ in $X_{n}$ with at most 8 sides, which is $6 \delta_{0}$-thin. Hence the previous argument gives the same result with even sharper constant.

Finally, let us assume that only one side of $T$ intersects $X_{n}$. Then $z$ belongs to some $\left[b_{1}, b_{2}\right]$, and the same inequality holds.

Consequently, $X$ is $\delta$-thin with $\delta:=20 \delta_{0}+\max \left\{c_{1}+c_{2} / 2, c_{2}\right\}$.

The same proof of Theorem 3.1 gives sharper constants in some particular cases.

Corollary 3.2. Under the hypothesis of Theorem 3.1, we have that:

(1) We can take $\delta:=\max \left\{2 \delta_{0}+c_{2}, 6 \delta_{0}+c_{2} / 2,3 c_{2} / 2\right\}$, if $B_{n}=\varnothing$ for every $n \in \Lambda$.

(2) We can take $\delta:=4 \delta_{0}+c_{1}$, if $A_{n}=\varnothing$ for every $n \in \Lambda$.

\section{Background in Riemann surfaces}

We collect here some definitions concerning Riemann surfaces.

An open non-exceptional Riemann surface (or a non-exceptional Riemann surface without boundary) $S$ is a Riemann surface whose universal covering space is the unit disk $\mathbf{D}=\{z \in \mathbf{C}:|z|<1\}$, endowed with its Poincaré metric, i.e. the metric obtained by projecting the Poincaré 
metric of the unit disk

$$
d s=\lambda_{\mathbf{D}}(z)|d z|=\frac{2|d z|}{1-|z|^{2}},
$$

or, equivalently, the upper half plane $\mathbf{U}=\{z \in \mathbf{C}: \operatorname{Im} z>0\}$, with the metric $d s=\lambda_{\mathbf{U}}(z)|d z|=|d z| / \operatorname{Im} z$. Observe that, with this definition, every compact non-exceptional Riemann surface without boundary is open. With this metric, $S$ is a complete Riemannian manifold with constant curvature -1 ; therefore $S$ is a geodesic metric space. The only Riemann surfaces which are left out are the sphere, the plane, the punctured plane and the tori. It is easy to study hyperbolicity of these particular cases.

It is well-known (see e.g. [An, p. 100], [B, p. 131], [JS, p. 227], [N, p. 18]) that

$$
\begin{gathered}
d_{\mathbf{D}}(0, z)=\log \frac{1+|z|}{1-|z|}=2 \operatorname{Arctanh}|z|, \\
\sinh ^{2} \frac{d_{\mathbf{U}}(z, w)}{2}=\frac{|z-w|^{2}}{4 \operatorname{Im} z \operatorname{Im} w} .
\end{gathered}
$$

A collar in $S$ about a simple closed geodesic $\gamma$ is a doubly connected domain in $S$ "bounded" by two Jordan curves (called the boundary curves of the collar) orthogonal to the pencil of geodesics emanating from $\gamma$; such a collar is equal to $\left\{p \in S: d_{S}(p, \gamma)<d\right\}$, for some positive constant $d$. The constant $d$ is called the width of the collar. The Collar Lemma $[\mathbf{R}]$ says that there exists a collar of $\gamma$ of width $d$, for every $0<d \leq d_{0}$, where $\cosh d_{0}=\operatorname{coth}\left(L_{S}(\gamma) / 2\right)$, or similarly $\sinh d_{0}=\operatorname{cosech}\left(L_{S}(\gamma) / 2\right)$.

As we remarked after Lemma 3.2, every closed geodesic is a local geodesic, but not a geodesic; however, since there is no possible confusion, we call it closed geodesic instead of closed local geodesic.

A puncture in a non-exceptional Riemann surface is a doubly connected end in which we can find homotopically non-trivial curves with arbitrarily small length. A puncture is an isolated point in $\partial S$ in the case that $S \subset \mathbf{C}$. We can think of a puncture as a boundary geodesic of zero length.

We say that $S$ is a bordered non-exceptional Riemann surface (or a non-exceptional Riemann surface with boundary) if it can be obtained by deleting an open set $V$ of an open non-exceptional Riemann surface $R$, with $d_{S}:=\left.d_{R}\right|_{S}$ (recall Definition 2.6). Any such surface $S$ is 
a bordered orientable Riemannian manifold of dimension 2 and its Riemannian metric has constant negative curvature -1 . It is not difficult to see that if any ball in $R$ intersects at most a finite number of connected components of $V$, and the boundary of $S$ is locally Lipschitz, then $S$ is a geodesic metric space.

A funnel is a bordered non-exceptional Riemann surface which is topologically a cylinder and whose boundary is a simple closed geodesic. Given a positive number $a$, there is a unique (up to conformal mapping) funnel such that its boundary curve has length $a$. Every funnel is conformally equivalent, for some $\beta>1$, to the subset $\{z \in \mathbf{C}: 1 \leq|z|<\beta\}$ of the annulus $\{z \in \mathbf{C}: 1 / \beta<|z|<\beta\}$.

Every doubly connected end of an open non-exceptional Riemann surface is a puncture (if there are homotopically non-trivial curves with arbitrary small length) or a funnel (in other case).

A $Y$-piece is a bordered non-exceptional Riemann surface which is conformally equivalent to a sphere minus three open disks and whose boundary curves are simple closed geodesics (and then it is triply connected). Given three positive numbers $a, b, c$, there is a unique (up to conformal mapping) $Y$-piece such that their boundary curves have lengths $a, b, c$ (see e.g. [Ra, p. 410]). They are a standard tool for constructing Riemann surfaces. A clear description of these $Y$-pieces and their use are given in [Bu, Chapter 1] and [C, Chapter X.3].

A generalized $Y$-piece is a non-exceptional Riemann surface (with or without boundary) which is conformally equivalent to a sphere without $n$ open disks and $m$ points, with integers $n, m \geq 0$ such that $n+m=3$, the $n$ boundary curves are simple closed geodesics and the $m$ deleted points are punctures. Observe that a generalized $Y$-piece is topologically the union of a $Y$-piece and $m$ cylinders.

\section{Results in Riemann surfaces}

Although one should expect Gromov hyperbolicity in non-exceptional Riemann surfaces due to its constant curvature -1 , this turns out to be untrue in general, since topological obstacles can impede it: for instance, the two-dimensional jungle-gym (a $\mathbf{Z}^{2}$-covering of a torus with genus two) is not hyperbolic.

In [RT2] we prove that there is no inclusion relationship between hyperbolic Riemann surfaces and the usual classes of Riemann surfaces, such as $O_{G}, O_{H P}, O_{H B}, O_{H D}$, surfaces with linear isoperimetric inequality, or the complements of these classes (even in the case of plane 
domains). This fact shows that the study of hyperbolic Riemann surfaces is more complicated and interesting than one might think at first sight. One can find other results on hyperbolicity of Riemann surfaces in [RT1], [RT2], [PRT1] and [PRT2].

The main result in this paper is Theorem 5.1, which allows us to reduce drastically the triangles in which we have to check the Rips condition in Riemann surfaces. In [FR1, Lemma 1.2] it is proved that in order to check the linear isoperimetric inequality in a Riemann surface it is enough to deal with domains whose boundary is a finite union of simple closed geodesics; this fact is interesting by itself and has important consequences, as the stability of linear isoperimetric inequality under quasiconformal maps (see [FR1, Theorem 1]), and the equivalence of linear isoperimetric inequality and Poincaré's inequality (see [FR1, Theorem 2]). Here we prove that if the triangles contained in simple closed geodesics of a Riemann surface $S$ satisfy the Rips condition, then $S$ is hyperbolic (see Theorem 5.1).

The results in this section give many examples of hyperbolic Riemann surfaces, and provide criteria in order to decide whether a Riemann surface is hyperbolic or not.

Definition 5.1. By a simply connected polygon in a non-exceptional Riemann surface we mean a polygon isometric to a polygon in the Poincaré disk. We say that two sides in a polygon are disjoint if their interiors are disjoint.

We collect in the following lemmas some technical results which we need in order to clarify the proof of Theorem 5.1 .

Lemma 5.1. Let us consider a simply connected locally geodesic quadrilateral in a non-exceptional Riemann surface $S$ with pairwise disjoint sides $A, C, B$ and $\eta$, of lengths $a, c, b$ and $l_{0}$, respectively. Let us assume also that $C$ meets orthogonally the sides $A$ and $B$. We have that:

(1) $\cosh l_{0}=\cosh a \cosh b \cosh c-\sinh a \sinh b$.

(2) Let us fix $c_{0}>0$. If $c \geq c_{0}$, then $a+b+c-c_{1} \leq l_{0}$, with $c_{1}:=3 \log 2-2 \log \left(1-e^{-c_{0}}\right)$.

(3) If $c_{0}:=\log (5+2 \sqrt{6})$, then $c_{1}=c_{0}$.

Remark. It is clear by the triangle inequality that $l_{0} \leq a+b+c$. 
Proof: Since the quadrilateral is simply connected, we can assume that it is contained in the unit disk D. Part (1) can be found in [F, p. 88].

We show part (2). Let us observe that the function $f(t):=2(\cosh t-$ 1) $e^{-t}=\left(1-e^{-t}\right)^{2}$ is increasing in $[0, \infty)$. Then $f(c) \geq f\left(c_{0}\right)=(1-$ $\left.e^{-c_{0}}\right)^{2}$, for $c \geq c_{0}$, i.e. $\cosh c-1 \geq \frac{1}{2} f\left(c_{0}\right) e^{c}$. Consequently, if $c \geq c_{0}$,

$$
\begin{aligned}
& e^{l_{0}} \geq \cosh l_{0}=\cosh a \cosh b \cosh c-\sinh a \sinh b \\
& \quad \geq(\cosh c-1) \cosh a \cosh b \geq \frac{1}{8} f\left(c_{0}\right) e^{a+b+c},
\end{aligned}
$$

and we deduce $l_{0} \geq a+b+c+\log \frac{1}{8}\left(1-e^{-c_{0}}\right)^{2}=a+b+c-c_{1}$.

A direct computation gives (3).

Lemma 5.2. Let us consider a simply connected self-intersecting locally geodesic quadrilateral in a non-exceptional Riemann surface $S$ with sides $A, C, B$ and $\eta$, of lengths $a, c, b$ and $l_{0}$, respectively. Let us assume also that $C$ meets orthogonally the sides $A$ and $B$. If $\eta$ and $C$ are not disjoint, then we have that:

(1) $\cosh l_{0}=\cosh a \cosh b \cosh c+\sinh a \sinh b$.

(2) $a+b+c-3 \log 2 \leq l_{0}$.

Proof: Since the quadrilateral is simply connected, we can assume that it is contained in the unit disk D. Part (1) can be found in $[\mathbf{F}$, p. 89].

We show part (2). The inequality is a consequence of

$$
\begin{aligned}
& e^{l_{0}} \geq \cosh l_{0}=\cosh a \cosh b \cosh c+\sinh a \sinh b \\
& \quad \geq \cosh a \cosh b \cosh c \geq \frac{1}{8} e^{a+b+c} .
\end{aligned}
$$

Lemma 5.3. Let us consider $c_{0}>0$ and a simply connected locally geodesic quadrilateral $Q$ in a non-exceptional Riemann surface $S$ with pairwise disjoint sides $A, C, B$ and $\eta$, of lengths $a, c, b$ and $l_{0}$, respectively. Let us assume also that $C$ meets orthogonally the sides $A$ and $B$. If $c \geq c_{0}$, then we have that

$$
d(z, \eta)<c_{2}:=\operatorname{Arcsinh} \frac{e^{c_{0}}+1}{e^{c_{0}}-1}=\operatorname{Arcsinh}\left(\operatorname{cotanh} \frac{c_{0}}{2}\right),
$$

for every $z \in A \cup B \cup C$. 
Proof: Since $Q$ is simply connected, we can assume that it is contained in the upper half plane $\mathbf{U}$. Without loss of generality we can assume that $Q$ is the quadrilateral with vertices $i, i t, i e^{-i \theta}, i e^{-i \phi} t$, with $0<\theta$, $\phi<\pi / 2$ and $t=e^{c} \geq e^{c_{0}}$.

It is clear that $d(z, \eta) \leq \max \{d(i, \eta), d(i t, \eta)\}$. Without loss of generality we can assume that $d(i, \eta)=\max \{d(i, \eta), d(i t, \eta)\}$ (if it is not the case, we can change the roles of $\theta$ and $\phi)$.

It is obvious that $d(i, \eta)$ is less than the distance of $i$ to the geodesic $\eta_{0}$ joining 1 and $t$.

The Möbius transformation $T z:=(z-t) /(z-1)$ maps $\eta_{0}$ onto the imaginary half-axis $I$, and $T i=(t+1+i(t-1)) / 2$. A computation gives (see e.g. [B, p. 162])

$$
d(z, \eta)<d\left(i, \eta_{0}\right)=d(T i, I)=\operatorname{Arcsinh} \frac{t+1}{t-1} \leq \operatorname{Arcsinh} \frac{e^{c_{0}}+1}{e^{c_{0}}-1},
$$

since $t \geq e^{c_{0}}$

Lemma 5.4. Let us consider the annulus $A_{l}$ such that its simple closed geodesic has length $l$; we denote by $A_{0}$ the limit case $A_{0}:=\mathbf{D}^{*}:=$ $\mathbf{D} \backslash\{0\}$. Then $A_{l}$ is $\delta(l)$-thin for any $l \geq 0$, where $\delta(l):=\max \{l+$ $2 \log (1+\sqrt{2}), d(l)+3 \log (1+\sqrt{2}), d(l) / 2+6 \log (1+\sqrt{2})\}$, with $d(l):=$ $\operatorname{Arcsinh}(\sinh (l / 2) \operatorname{cotanh}(l / 6))$ if $l>0$ and $d(0):=\operatorname{Arcsinh} 3$. In particular, $\delta(0):=\frac{1}{2} \operatorname{Arcsinh} 3+6 \log (1+\sqrt{2})<6.1975$.

Proof: Let us consider a geodesic triangle $T=\{a, b, c\}$ in $A_{l}$. If $T$ is homotopic to a point, then it is the boundary of a simply connected closed set $E$, and consequently $E$, with its intrinsic distance, is isometric to some subset of $\mathbf{D}$; this implies that $T$ is $\delta_{0}$-thin, with $\delta_{0}:=\log (1+\sqrt{2})$, since $\mathbf{D}$ is $\delta_{0}$-thin (see [An, p. 130]). Then we can assume that $T$ is freely homotopic to the simple closed geodesic $g$, if $l>0$, or to the puncture, if $l=0$.

Let us assume first that $l>0$ and $T \cap g \neq \varnothing$. We denote by $F^{1}$ and $F^{2}$ the two funnels whose union is $A_{l}$ (the closures of the two connected components of $A_{l} \backslash g$ ).

Let us observe that the funnels are geodesically convex (every geodesic connecting two points of the funnel is contained in the funnel). Hence, without loss of generality we can assume that $a$ is in the interior of $F^{1}$ and $b, c$ are in the interior of $F^{2}$ (the case in which there is some vertex in $g$ is easier). We define $B:=[a, b] \cap g$ and $C:=[a, c] \cap g$. There are two local geodesics $g_{1}, g_{2} \subset g$ joining $B$ and $C$; let us observe that $L_{A_{l}}\left(g_{i}\right) \leq l$.

Let us consider the triangle $T_{1}=\{a, B, C\}$, where we choose as $[B, C]$ the local geodesic $g_{i} \subset g$ such that $[a, B] \cup[B, C] \cup[C, a]$ is homotopic 
to a point; since $T_{1}$ is homotopic to a point, the above argument implies that $T_{1}$ is $\delta_{0}$-thin. Given $x \in[a, B]$ there is some $y \in[a, C] \cup[B, C]$ with $d_{A_{l}}(x, y) \leq \delta_{0}$; if $y \in[a, C]$, then $d_{A_{l}}(x,[a, C]) \leq \delta_{0}$; if $y \in[B, C]$, we have $d_{A_{l}}(x,[a, C]) \leq d_{A_{l}}(x, y)+d_{A_{l}}(y, C) \leq \delta_{0}+l$. If $x \in[a, C]$, we obtain a similar result.

Let us consider the quadrilateral $Q_{1}=\{b, c, C, B\}$, where we choose as $[B, C]$ the local geodesic $g_{i} \subset g$ such that $[b, c] \cup[c, C] \cup[C, B] \cup[B, b]$ is homotopic to a point; since $Q_{1}$ is homotopic to a point, the above argument implies that $Q_{1}$ is $2 \delta_{0}$-thin. In a similar way to the case of $T_{1}$, given any point in $T \cap Q_{1}$ there is a point $y \in T \cap Q_{1}$ (in other side of $T$ ) with $d_{A_{l}}(x, y) \leq 2 \delta_{0}+l$. Then $T$ is $\left(2 \delta_{0}+l\right)$-thin.

Let us assume now that $l>0$ and $T \cap g=\varnothing$. Next, we find an upper bound for $d_{A_{l}}(T, g)$. Given a point $w$ of $T$, we denote by $w_{0}$ the point in $g$ with $d_{A_{l}}\left(w, w_{0}\right)=d_{A_{l}}(w, g)$. If $T=\{a, b, c\}$, we have that $d_{A_{l}}\left(a_{0}, b_{0}\right)+d_{A_{l}}\left(b_{0}, c_{0}\right)+d_{A_{l}}\left(c_{0}, a_{0}\right)=l$. Hence, without loss of generality we can assume that $d_{A_{l}}\left(a_{0}, b_{0}\right) \geq l / 3$. Let us consider the point $x \in[a, b]$ with $d_{A_{l}}(x, g)=d_{A_{l}}([a, b], g)$. We consider first the case $x \in(a, b)$. We can assume that $t:=d_{A_{l}}\left(a_{0}, x_{0}\right) \geq l / 6$.

We consider now the geodesic quadrilateral $Q:=\left\{a, a_{0}, x_{0}, x\right\}$ with three right angles (known as Lambert quadrilateral). If $s:=d_{A_{l}}\left(x_{0}, x\right)$ and $\phi$ is the angle of $\left[a, a_{0}\right]$ and $[a, x]$ in $a$, the trigonometric formulas give $\sinh s \sinh t=\cos \phi$ (see e.g. [B, p. 157], [C, p. 263]). Then

$$
\sinh s=\frac{\cos \phi}{\sinh t}<\frac{1}{\sinh t} \leq \frac{1}{\sinh (l / 6)}
$$

Therefore, we have that

$$
d_{A_{l}}(T, g)<\operatorname{Arcsinh} \frac{1}{\sinh (l / 6)}
$$

If $x=a$ or $x=b$, a similar argument with $t:=d_{A_{l}}\left(a_{0}, b_{0}\right)$ gives $\sinh s \sinh t<1$, and we obtain $\sinh s<1 / \sinh (l / 3)$, which also implies (5.1).

Without loss of generality we can assume that $d_{A_{l}}(T, g)=d_{A_{l}}(x, g)=$ $d_{A_{l}}\left(x, x_{0}\right)=s$. Let us consider the local geodesic $g_{x}$ starting and finishing in $x$, which is freely homotopic to $g$. We consider first the case $x \in(a, b)$. We denote by $2 d_{x}$ the length of $g_{x}$ and by $y$ the point in $g_{x}$ at distance $d_{x}$ of $x$.

We consider the geodesic quadrilateral $R:=\left\{x, x_{0}, y_{0}, y\right\}$ with three right angles. Since $d_{A_{l}}\left(x_{0}, y_{0}\right)=l / 2$, the trigonometric formulas give 
(see e.g. [F, p. 88])

$$
\begin{aligned}
\sinh d_{x} & =\sinh (l / 2) \cosh s=\sinh (l / 2) \sqrt{1+\sinh ^{2} s} \\
& <\sinh (l / 2) \sqrt{1+\operatorname{cosech}^{2}(l / 6)}=\sinh (l / 2) \operatorname{cotanh}(l / 6) .
\end{aligned}
$$

Let us assume now that $l=0$, i.e. that we deal with the case $A_{0}=\mathbf{D}^{*}$; then $T$ is freely homotopic to the puncture. We consider the universal covering map $\pi: \mathbf{U} \longrightarrow \mathbf{D}^{*}$, given by $\pi(z)=\exp (2 \pi i z)$. It is clear that $\pi$ maps bijectively $\left.U_{0}:=\{z \in \mathbf{U}: 0 \leq \Re z<1\}\right)$ in $\mathbf{D}^{*}$. Without loss of generality we can assume that $\pi\left(z_{1}\right)=a, \pi\left(z_{2}\right)=b$ and $\pi\left(z_{3}\right)=c$, with $\Re z_{1}=0$ and $1 / 3 \leq \Re z_{2} \leq \Re z_{3}<1$. Since $\Re\left(z_{2}-z_{1}\right) \geq 1 / 3$, there exists a point $z \in\left[z_{1}, z_{2}\right]$ with $\Im z>1 / 6$; then $\max \{\Im z: \pi(z) \in T\}>1 / 6$. We denote by $z_{0}$ a point of $U_{0}$ in which this maximum is attained.

Let us consider the local geodesic $g_{0}$ in $\mathbf{D}^{*}$ starting and finishing in $\pi\left(z_{0}\right)$, which is freely homotopic to the puncture; if we denote by $2 d_{\pi\left(z_{0}\right)}$ the length of $g_{0},(4.1)$ gives that

$$
\sinh ^{2} d_{\pi\left(z_{0}\right)}=\sinh ^{2} \frac{d_{\mathbf{U}}\left(z_{0}, 1+z_{0}\right)}{2}<\sinh ^{2} \frac{d_{\mathbf{U}}(i / 6,1+i / 6)}{2}=9
$$

and consequently $d_{\pi\left(z_{0}\right)}<\operatorname{Arcsinh} 3$.

Recall that $d(l):=\operatorname{Arcsinh}(\sinh (l / 2) \operatorname{cotanh}(l / 6))$ if $l>0$ and $d(0):=$ Arcsinh 3. Then there exists a point $p \in T$ such that the local geodesic $g_{p}$ in $A_{l}$ starting and finishing in $p$, which is freely homotopic to $g$ or to the puncture, has length $2 d_{p}<2 d(l)$.

Let us assume first that $p$ is not a vertex of $T$; without loss of generality we can assume also that $p \in[a, c]$. Since $g_{p}$ is freely homotopic to $T$, we have a geodesic pentagon $P^{\prime}:=\left\{a^{\prime}, b^{\prime}, c^{\prime}, p_{1}^{\prime}, p_{2}^{\prime}\right\}$ in $\mathbf{D}$, which is isometric to the pentagon $P$ made of $[a, b],[b, c],[c, p], g_{p}$ and $[p, a]$, if we identify $p_{1}^{\prime}$ with $p_{2}^{\prime}$ (we have chosen $P^{\prime}$ such that $d_{\mathbf{D}}\left(a^{\prime}, b^{\prime}\right)=d_{A_{l}}(a, b)$, $d_{\mathbf{D}}\left(b^{\prime}, c^{\prime}\right)=d_{A_{l}}(b, c), d_{\mathbf{D}}\left(c^{\prime}, p_{1}^{\prime}\right)=d_{A_{l}}(c, p), d_{\mathbf{D}}\left(p_{1}^{\prime}, p_{2}^{\prime}\right)=L_{A_{l}}\left(g_{p}\right)$ and $\left.d_{\mathbf{D}}\left(p_{2}^{\prime}, a^{\prime}\right)=d_{A_{l}}(p, a)\right)$.

It is clear that if $x^{\prime}, y^{\prime}$, are the corresponding points in $P^{\prime}$ to the points $x, y \in P$, we have $d_{A_{l}}(x, y) \leq d_{\mathbf{D}}\left(x^{\prime}, y^{\prime}\right)$.

Now we use a similar argument to the one in the proof of Theorem 3.1.

Since $P^{\prime}$ is a geodesic pentagon in $\mathbf{D}$, we have that it is $3 \delta_{0}$-thin. Let us consider the point $\alpha_{1}^{\prime}$ in the oriented geodesic $\left[p_{1}^{\prime}, c^{\prime}\right]$, defined by $\alpha_{1}^{\prime}:=\max \left\{z \in\left[p_{1}^{\prime}, c^{\prime}\right]: d_{\mathbf{D}}\left(z,\left[p_{1}^{\prime}, p_{2}^{\prime}\right]\right) \leq 3 \delta_{0}\right\}$, and the point $\alpha_{2}^{\prime}$ in the oriented geodesic $\left[p_{2}^{\prime}, a^{\prime}\right]$, defined by $\alpha_{2}^{\prime}:=\max \left\{z \in\left[p_{2}^{\prime}, a^{\prime}\right]\right.$ : $\left.d_{\mathbf{D}}\left(z,\left[p_{1}^{\prime}, p_{2}^{\prime}\right]\right) \leq 3 \delta_{0}\right\}$. 
If $\alpha_{j}$ is the corresponding point in $P$ to $\alpha_{j}^{\prime}$, we have that $L_{A_{l}}\left(\left[\alpha_{1}, \alpha_{2}\right]\right)=$ $d_{A_{l}}\left(\alpha_{1}, \alpha_{2}\right) \leq 6 \delta_{0}+d(l)$, since $d_{A_{l}}\left(\alpha_{j}, g_{p}\right) \leq 3 \delta_{0}$ and $\operatorname{diam}_{A_{l}}\left(g_{p}\right) \leq d_{p}<$ $d(l)$.

We define now $\beta_{1}^{\prime}:=\max \left(\left\{\alpha_{1}^{\prime}\right\} \cup\left\{z \in\left[p_{1}^{\prime}, c^{\prime}\right]: d_{\mathbf{D}}\left(z,\left[p_{2}^{\prime}, a^{\prime}\right]\right) \leq 3 \delta_{0}\right\}\right)$, $\beta_{2}^{\prime}:=\max \left(\left\{\alpha_{2}^{\prime}\right\} \cup\left\{z \in\left[p_{2}^{\prime}, a^{\prime}\right]: d_{\mathbf{D}}\left(z,\left[p_{1}^{\prime}, c^{\prime}\right]\right) \leq 3 \delta_{0}\right\}\right)$. Let us denote by $\beta_{j}$ the corresponding point in $P$ to $\beta_{j}^{\prime}$.

If $\beta_{1} \neq \alpha_{1}$, then $d_{A_{l}}\left(\beta_{1}, p\right)=L_{A_{l}}\left(\left[\beta_{1}, p\right]\right)=d_{A_{l}}\left(\beta_{1},[p, a]\right) \leq 3 \delta_{0}$; in a similar way, if $\beta_{2} \neq \alpha_{2}$, then $d_{A_{l}}\left(\beta_{2}, p\right) \leq 3 \delta_{0}$. We consider now the next four possibilities:

- If $\beta_{1}=\alpha_{1}$ and $\beta_{2}=\alpha_{2}$, we have seen that $d_{A_{l}}\left(\beta_{1}, \beta_{2}\right) \leq 6 \delta_{0}+d(l)$.

- If $\beta_{1} \neq \alpha_{1}$ and $\beta_{2} \neq \alpha_{2}$, then $d_{A_{l}}\left(\beta_{1}, \beta_{2}\right) \leq d_{A_{l}}\left(\beta_{1}, p\right)+d_{A_{l}}\left(p, \beta_{2}\right) \leq$ $6 \delta_{0}$.

- If $\beta_{1} \neq \alpha_{1}$ and $\beta_{2}=\alpha_{2}$, then there is a point $z_{0} \in\left[p_{1}^{\prime}, p_{2}^{\prime}\right]$ with $d_{\mathbf{D}}\left(\beta_{2}^{\prime}, z_{0}\right) \leq 3 \delta_{0}$; since there is some $p_{i}^{\prime}$ with $d_{\mathbf{D}}\left(p_{i}^{\prime}, z_{0}\right) \leq d(l)$, we obtain that $d_{A_{l}}\left(\beta_{1}, \beta_{2}\right) \leq d_{\mathbf{D}}\left(\beta_{1}^{\prime}, \beta_{2}^{\prime}\right) \leq d_{\mathbf{D}}\left(\beta_{1}^{\prime}, p_{i}^{\prime}\right)+d_{\mathbf{D}}\left(p_{i}^{\prime}, z_{0}\right)+$ $d_{\mathbf{D}}\left(z_{0}, \beta_{2}^{\prime}\right) \leq 6 \delta_{0}+d(l)$.

- If $\beta_{1}=\alpha_{1}$ and $\beta_{2} \neq \alpha_{2}$, we obtain in a similar way that $d_{A_{l}}\left(\beta_{1}, \beta_{2}\right) \leq$ $6 \delta_{0}+d(l)$.

Therefore, in the four situations we have $d_{A_{l}}\left(\beta_{1}, \beta_{2}\right) \leq 6 \delta_{0}+d(l)$. If $x \in\left[\beta_{1}, c\right] \cup\left[\beta_{2}, a\right]$, then $d_{A_{l}}(x,[a, b] \cup[b, c]) \leq 3 \delta_{0}$. If $x \in\left[\beta_{1}, \beta_{2}\right]$, we can take $\beta_{i}$ with $d_{A_{l}}\left(x, \beta_{i}\right) \leq 3 \delta_{0}+d(l) / 2$; since $d_{A_{l}}\left(\beta_{i},[a, b] \cup[b, c]\right) \leq 3 \delta_{0}$, we obtain $d_{A_{l}}(x,[a, b] \cup[b, c]) \leq 6 \delta_{0}+d(l) / 2$.

If $x \in[a, b]$, there exists a point $y^{\prime} \in P^{\prime} \backslash\left(a^{\prime}, b^{\prime}\right)$ with $d_{\mathbf{D}}\left(x^{\prime}, y^{\prime}\right) \leq 3 \delta_{0}$. If $y^{\prime} \notin\left[p_{1}^{\prime}, p_{2}^{\prime}\right]$, then $d_{A_{l}}(x,[b, c] \cup[c, a]) \leq 3 \delta_{0}$. If $y^{\prime} \in\left[p_{1}^{\prime}, p_{2}^{\prime}\right]$, there is $p_{i}^{\prime}$ with $d_{\mathbf{D}}\left(y^{\prime}, p_{i}^{\prime}\right) \leq d(l)$, and hence $d_{\mathbf{D}}\left(x^{\prime}, p_{i}^{\prime}\right) \leq 3 \delta_{0}+d(l)$. Since $p \in[a, c]$, we have that $d_{A_{l}}(x,[b, c] \cup[c, a]) \leq 3 \delta_{0}+d(l)$. A similar result is true if $x \in[b, c]$. These facts give that $T$ is $\max \left\{3 \delta_{0}+d(l), 6 \delta_{0}+d(l) / 2\right\}$-thin.

If $p$ is a vertex of $T$, the proof is easier since we construct a quadrilateral instead of a pentagon, and we do not need to split a side of $T$. This finishes the proof of Lemma 5.4.

The following is the main result of this paper; it allows one to check the Rips condition only for triangles contained in simple closed geodesics. We would like to remark the simplification that Theorem 5.1 means in the applications: Let us consider an annulus $A$ with simple closed geodesic $\gamma$. A generic triangle $T$ in $A$ is determined by the coordinates of three points, i.e., by six real coordinates; however, a generic triangle $T_{0}$ in the simple closed geodesic $\gamma$ is determined by three real coordinates. Therefore Theorem 5.1 is a remarkable improvement of Rips condition in the context of Riemann surfaces. 
Definition 5.2. By a $c_{0}$-triangle we mean a triangle with continuous injective $\left(1, c_{0}\right)$-quasigeodesic sides, with its arc-length parametrization.

We define the constants

$$
\begin{gathered}
c_{0}:=\log (5+2 \sqrt{6})<2.2925 \\
K:=2 \log (1+\sqrt{2})+\log (5+2 \sqrt{6})+\log \frac{\sqrt{6}+\sqrt{10}}{2}<5.0869 .
\end{gathered}
$$

Theorem 5.1. Let us consider a non-exceptional Riemann surface $S$ (with or without boundary); if $S$ has boundary, we also require that $\partial S$ is the union of local geodesics (closed or non-closed). Then $S$ is hyperbolic if and only if every $c_{0}$-triangle contained in a simple closed geodesic in $S$ is $\delta_{0}$-thin.

Furthermore, if every $c_{0}$-triangle contained in a simple closed geodesic in $S$ is $\delta_{0}$-thin, then $S$ is $\delta$-thin, with $\delta=\max \left\{\delta\left(4 c_{0}\right), \delta_{0}+K\right\}$, where $\delta(t)$ is the constant in Lemma 5.4 (it verifies $\delta\left(4 c_{0}\right)<10.9325$ ).

Remarks. 1. Although one can think of quasigeodesic triangles as an artificial technical device, the example after the proof of Theorem 5.1 shows that they are essential.

2. Even though this theorem reduces drastically the triangles in which we have to check the Rips condition, we must "pay" for it by working with quasigeodesic triangles; however the situation is advantageous since the class of quasigeodesics that we need is very restrictive: recall that we only consider continuous injective $\left(1, c_{0}\right)$-quasigeodesics, and Lemma 3.2 gives a bound of its length which will be good enough in the applications (see Theorem 5.3 and Corollaries 5.2 and 5.3).

Proof: The heart of the proof of Theorem 5.1 is to relate any geodesic triangle $T$ in $S$ with a $c_{0}$-triangle contained in a simple closed geodesic $\gamma$ in $S$. In some way, we can consider $T$ and $\gamma$ as "subsets" of the annulus $A_{l}$ (with $l:=L_{S}(\gamma)$ ). The geodesic triangles in the simple closed geodesic of $A_{l}$ are $(l / 4)$-thin, and this value is sharp (it is enough to consider a triangle with sides of lengths $l / 4, l / 4$ and $l / 2$ ). However, the problem in a general Riemann surface is more difficult (and recall that we can find simple closed geodesics arbitrarily long). Therefore, if $l$ is big we need a narrow metric relationship between $T$ and $\gamma$.

If $S$ is hyperbolic, Lemma 3.3 guarantees that every $c_{0}$-triangle in $S$ is $\delta_{0}$-thin.

Let us assume that every $c_{0}$-triangle contained in a simple closed geodesic in $S$ is $\delta_{0}$-thin. First, we want to remark that if $S$ has boundary, 
the hypothesis on $\partial S$ gives that it is the union of pairwise disjoint simple local geodesics (closed or non-closed).

In this case, we can construct an open non-exceptional Riemann surface $R$ by pasting to $S$ a funnel in each simple closed geodesic, and a half-disk in each non-closed simple geodesic.

Since $S$ is geodesically convex in $R$ (every geodesic connecting two points of $S$ is contained in $S)$, then $d_{R}(z, w)=d_{S}(z, w)$ for every $z, w \in S$, and any simple closed geodesic in $R$ is contained in $S$.

Let us consider a geodesic triangle $T$ in $S$. By Lemma 2.1 in [RT1], we can assume that $T$ is a simple closed curve.

We have three possibilities: $T$ is homotopic to a point, $T$ is homotopic to a puncture, or $T$ is freely homotopic to a simple closed geodesic in $S$. This is well known if $S$ has no boundary; if $S$ has boundary, it is enough to apply the result to $R$, since $R$ has not additional topological obstacles (the fundamental groups of $S$ and $R$ are isomorphic).

If $T$ is homotopic to a point, then it is the boundary of a simply connected closed set $E$, and consequently $E$, with its intrinsic distance, is isometric to some subset of $\mathbf{D}$; this implies that $T$ is $\log (1+\sqrt{2})$-thin, since $\mathbf{D}$ is $\log (1+\sqrt{2})$-thin (see [An, p. 130]).

If $T$ is homotopic to a puncture, then it is the boundary of a closed doubly connected set, which is, with its intrinsic distance, isometric to some subset of $\mathbf{D}^{*}:=\mathbf{D} \backslash\{0\}$; this implies that $T$ is $\delta(0)$-thin, with $\delta(0)$ the constant in Lemma 5.4. Since every geodesic triangle in $\mathbf{D}$ is isometric to some geodesic triangle in $\mathbf{D}^{*}$, we have that $\log (1+\sqrt{2}) \leq \delta(0)$.

In other case, $T$ is freely homotopic to a simple closed geodesic $\gamma$ in $S$.

If $L(\gamma)<4 c_{0}$, let us consider the annulus $A_{L(\gamma)}$ with a simple closed geodesic $g$ of length $L(\gamma)$. We have that $A_{L(\gamma)}$ is $\delta(L(\gamma))$-thin, with $\delta(L(\gamma))$ the constant in Lemma 5.4. Since

$$
d=d(l)=\operatorname{Arcsinh}\left(\frac{\sinh (l / 2)}{\sinh (l / 6)} \cosh (l / 6)\right),
$$

if $l>0$ and $d(0)=\lim _{l \rightarrow 0} d(l)$, we have that $d=d(l)$ is an increasing function for $l \geq 0$; then we also have that $\delta(0) \leq \delta(L(\gamma))<\delta\left(4 c_{0}\right)$, with

$$
\begin{aligned}
\delta\left(4 c_{0}\right)= & \max \left\{4 c_{0}+2 \log (1+\sqrt{2}),\right. \\
& \operatorname{Arcsinh}\left(\sinh \left(2 c_{0}\right) \operatorname{cotanh}\left(2 c_{0} / 3\right)\right)+3 \log (1+\sqrt{2}), \\
& \left.\frac{1}{2} \operatorname{Arcsinh}\left(\sinh \left(2 c_{0}\right) \operatorname{cotanh}\left(2 c_{0} / 3\right)\right)+6 \log (1+\sqrt{2})\right\} \\
= & 4 c_{0}+2 \log (1+\sqrt{2})<10.9325 .
\end{aligned}
$$


In this case, the closed set in $S$ bounded by $T$ and $\gamma$ is, with its intrinsic distance, isometric to a set in $A_{L(\gamma)}$, bounded by $g$ and a triangle $T_{0}$. These facts give that $T$ is $\delta\left(4 c_{0}\right)$-thin.

We consider now the case $L(\gamma) \geq 4 c_{0}$.

First, we assume that $\gamma \cap T=\varnothing$. If $\eta$ is a side of $T$, we associate to it two curves $\eta^{\prime}, \eta^{\prime \prime}$, in the following way. We consider a simply connected locally geodesic quadrilateral $Q$ in $S$ with pairwise disjoint sides $A, C, B$ and $\eta$, of lengths $a, c, b$ and $l_{0}$, respectively, with the following conditions: (i) $C \subset \gamma$, (ii) $C$ meets orthogonally the sides $A$ and $B . Q$ is uniquely determined by these conditions. If $c \geq c_{0}$, the $\operatorname{arc} \eta^{\prime}:=A \cup C \cup B$ is a continuous injective $\left(1, c_{0}\right)$-quasigeodesic with its arc-length parametrization by Lemmas 3.1 and 5.1. If $c<c_{0}$, we take $\eta^{\prime}:=\eta$, which is a geodesic. (Observe that we have $c<c_{0}$ for at most one side of $T$, since $L(\gamma) \geq 4 c_{0}$; in other case, $T$ would not be a geodesic triangle.) In both cases, we define $\eta^{\prime \prime}:=C \subset \gamma$. We have that $\eta^{\prime \prime}$ is always a continuous injective $\left(1, c_{0}\right)$-quasigeodesic with its arclength parametrization: this is clear if $c \geq c_{0}$ (since $\eta^{\prime \prime} \subset \eta^{\prime}$ ), and it is a consequence of Corollary 3.1 if $c<c_{0}$.

If $T$ is the union of the geodesics $\eta_{1}, \eta_{2}, \eta_{3}$, we consider the $\left(1, c_{0}\right)$ quasigeodesic triangle $T^{\prime}$ defined as the union of the $\left(1, c_{0}\right)$-quasigeodesics $\eta_{1}^{\prime}, \eta_{2}^{\prime}, \eta_{3}^{\prime}$. We consider also the $\left(1, c_{0}\right)$-quasigeodesic triangle $T^{\prime \prime} \subset \gamma$ defined as the union of the $\left(1, c_{0}\right)$-quasigeodesics $\eta_{1}^{\prime \prime}, \eta_{2}^{\prime \prime}, \eta_{3}^{\prime \prime}$.

By hypothesis, $T^{\prime \prime}$ is $\delta_{0}$-thin. We prove now that $T^{\prime}$ is $\delta_{1}$-thin, with $\delta_{1}:=\max \left\{\delta_{0}, 2 \log (1+\sqrt{2})\right\}+c_{0}$.

If $\eta_{i}^{\prime} \neq \eta_{i}$, for $i=1,2,3$, then $T^{\prime}$ is $\delta_{0}$-thin, since every point in $T^{\prime} \backslash T^{\prime \prime}$ belongs to two sides of $T^{\prime}$.

If it is not the case, there is only one $i$ with $\eta_{i}^{\prime}=\eta_{i}$; we can assume $\eta_{1}^{\prime}=\eta_{1}$. Let us consider the quadrilateral $Q_{1}$ with sides $A_{1}, C_{1}, B_{1}$ and $\eta_{1}$; we have that $L\left(C_{1}\right)<c_{0}$. Since $Q_{1}$ is simply connected, it is isometric to a quadrilateral in $\mathbf{D}$ which is $2 \log (1+\sqrt{2})$-thin.

Then for each $z \in \eta_{1}^{\prime}=\eta_{1}$, there exists $w \in A_{1} \cup C_{1} \cup B_{1}$ with $d(z, w) \leq 2 \log (1+\sqrt{2})$. If $w \in A_{1} \cup B_{1}$, then $d\left(z, \eta_{2}^{\prime} \cup \eta_{3}^{\prime}\right) \leq 2 \log (1+\sqrt{2})$. If $w \in C_{1}$, then there exists $w^{\prime} \in A_{1} \cup B_{1}$ with $d\left(w, w^{\prime}\right) \leq c_{0}$ (since $\left.L\left(C_{1}\right)<c_{0}\right)$, and we have $d\left(z, \eta_{2}^{\prime} \cup \eta_{3}^{\prime}\right) \leq 2 \log (1+\sqrt{2})+c_{0}$.

If $z \in \eta_{2}^{\prime}$, we consider three cases. If $z \in \eta_{2}^{\prime} \cap \gamma=\eta_{2}^{\prime \prime}$, then $d\left(z, \eta_{1}^{\prime} \cup\right.$ $\left.\eta_{3}^{\prime}\right) \leq d\left(z, \eta_{3}^{\prime \prime}\right) \leq d\left(z, \eta_{1}^{\prime \prime} \cup \eta_{3}^{\prime \prime}\right)+c_{0} \leq \delta_{0}+c_{0}$. If $z \in \eta_{2}^{\prime} \cap \eta_{3}^{\prime}$, then $d\left(z, \eta_{1}^{\prime} \cup \eta_{3}^{\prime}\right)=0$. In other case, $z \in A_{1} \cup B_{1}$ (we can assume that $A_{1} \subset \eta_{2}^{\prime}$ and $\left.B_{1} \subset \eta_{3}^{\prime}\right)$; then there exists $w \in B_{1} \cup C_{1} \cup \eta_{1}$ with $d(z, w) \leq$ $2 \log (1+\sqrt{2})$; since $L\left(C_{1}\right)<c_{0}$, there exists $w^{\prime} \in B_{1} \cup \eta_{1} \subset \eta_{3}^{\prime} \cup \eta_{1}^{\prime}$ with $d\left(w, w^{\prime}\right) \leq c_{0}$, and we have $d\left(z, \eta_{1}^{\prime} \cup \eta_{3}^{\prime}\right) \leq d\left(z, w^{\prime}\right) \leq 2 \log (1+\sqrt{2})+c_{0}$. 
Consequently, $T^{\prime}$ is $\delta_{1}$-thin, with $\delta_{1}:=\max \left\{\delta_{0}, 2 \log (1+\sqrt{2})\right\}+c_{0}$. The case $z \in \eta_{3}^{\prime}$ is similar to $z \in \eta_{2}^{\prime}$.

We show now that $T$ is $\delta_{2}$-thin, with

$$
\delta_{2}:=\delta_{1}+2 \log (1+\sqrt{2})+c_{2},
$$

and

$$
c_{2}:=\operatorname{Arcsinh}\left(\operatorname{cotanh} \frac{c_{0}}{2}\right)=\log \frac{\sqrt{6}+\sqrt{10}}{2} .
$$

Let us consider $x \in T$; we can assume that $x \in \eta_{1}$. If $\eta_{1} \neq \eta_{1}^{\prime}$, then $\eta_{1} \cup \eta_{1}^{\prime}$ is a simply connected geodesic quadrilateral, and therefore there exists $x^{\prime} \in \eta_{1}^{\prime}$ with $d\left(x, x^{\prime}\right) \leq 2 \log (1+\sqrt{2})$. If $\eta_{1}=\eta_{1}^{\prime}$, we take $x^{\prime}=x$. Then there exists $y^{\prime} \in \eta_{2}^{\prime} \cup \eta_{3}^{\prime}$ with $d\left(x^{\prime}, y^{\prime}\right) \leq \delta_{1}$; without loss of generality we can assume that $y^{\prime} \in \eta_{2}^{\prime}$. If $\eta_{2} \neq \eta_{2}^{\prime}$, Lemma 5.3 gives that there exists $y \in \eta_{2}$ with $d\left(y, y^{\prime}\right)<c_{2}$. If $\eta_{2}=\eta_{2}^{\prime}$, we take $y=y^{\prime}$. Consequently we have that $d(x, y)<\delta_{2}:=\delta_{1}+2 \log (1+\sqrt{2})+c_{2}=$ $\max \left\{\delta_{0}, 2 \log (1+\sqrt{2})\right\}+K$.

Therefore $T$ is $\delta$-thin, with $\delta:=\max \left\{\delta\left(4 c_{0}\right), \delta_{0}+K, 2 \log (1+\sqrt{2})+\right.$ $K\}=\max \left\{\delta\left(4 c_{0}\right), \delta_{0}+K\right\}$, since $\delta\left(4 c_{0}\right)>10>2 \log (1+\sqrt{2})+K$.

We assume now that $\gamma \cap T \neq \varnothing$.

If $\gamma \cap T$ has only one connected component, the same argument works.

If $\gamma \cap T$ has two connected components, the argument is similar, using now Lemma 5.2 instead of Lemma 5.1. The constant in this case is smaller, since $3 \log 2<c_{0}$.

The following example shows that the quasigeodesic triangle $T^{\prime \prime}$ in the proof of Theorem 5.1 does not need to be geodesic.

Example. There is a geodesic triangle $T$ in a triply connected Riemann surface $S_{0}$ such that $T^{\prime \prime}$ is not geodesic.

Given $x_{0}<\operatorname{Arcsinh} 1$, there exists $y>0$ with $\sinh \left(x_{0}+y\right)>\cosh y$. Then $\sinh (x+y)>\cosh y$ for any $x_{0} \leq x<\operatorname{Arcsinh} 1$, and consequently we can choose some $x<\operatorname{Arcsinh} 1 \operatorname{such}$ that $\sinh x \sinh (x+y)>\cosh y$.

If we define $\varepsilon:=\operatorname{Arcsinh}(1 / \sinh x)-x>0$, we have that $\sinh x \sinh (x+$ $\varepsilon)=1$. Let us consider a geodesic quadrilateral $V$ with three right angles and an angle equal to zero, such that the two finite sides have length $x$ and $x+\varepsilon$ (see e.g. [B, p. 157], [F, p. 89]). If we paste four quadrilaterals isometric to $V$, we obtain a generalized $Y$-piece $Y_{0}$ with two punctures and a simple closed geodesic $\gamma$ with $L(\gamma)=4(x+\varepsilon)$. We obtain $S_{0}$ by gluing $Y_{0}$ with a funnel $F$ whose simple closed geodesic has length $4(x+\varepsilon)$. 
Let us denote by $\mu_{0}$ the geodesic in $Y_{0}$ with $L\left(\mu_{0}\right)=2 x$, joining $\gamma$ with itself which is not homotopic to any curve contained in $\gamma$. We denote by $p^{\prime \prime}, q^{\prime \prime}$ the end points of $\mu_{0}$. Let us consider the non bounded geodesic $\mu$ in $S_{0}$ which contains $\mu_{0}$, and the two points $p, q \in \mu \cap F$ at distance $y$ of $\gamma$.

Let us define the triangle $T$ as the union of the two geodesics $\alpha, \beta$ in $F$ joining $p$ and $q$ (in fact, $T$ is a geodesic "bigon"). The length of the segment of $\mu$ between $p$ and $q$ is $2 x+2 y$; by [F, p. 88] we have $\sinh (L(\alpha) / 2)=\sinh (x+\varepsilon) \cosh y=\cosh y / \sinh x<\sinh (x+y)$; then we obtain $L(\alpha)<2 x+2 y$, and consequently $\alpha, \beta$ are in fact geodesics in $S_{0}$. However, $T^{\prime \prime}=\left\{p^{\prime \prime}, q^{\prime \prime}\right\}$ is contained in $\gamma$ and then $L\left(\alpha^{\prime \prime}\right)=$ $L\left(\beta^{\prime \prime}\right)=2 x+2 \varepsilon>2 x=L\left(\mu_{0}\right)$; hence $\alpha^{\prime \prime}, \beta^{\prime \prime}$ are not geodesics in $S_{0}$.

From now on we will obtain several consequences of Theorem 5.1.

Corollary 5.1. The annulus $A_{l}$ such that its simple closed geodesic has length $l \geq 4 c_{0}$ is $(l / 4+K)$-thin, with $K<5.0869$ the constant in Theorem 5.1. The same is true for each funnel of $A_{l}$.

Remark. This bound of the hyperbolicity constant for the annulus is asymptotically sharp: we have that the best thin constant of $A_{l}$ is greater than or equal to $l / 4$, since we have a geodesic triangle contained in the simple closed geodesic with sides of lengths $l / 2, l / 4, l / 4$.

Proof: Let us observe that the last part of the proof of Theorem 5.1 gives that $A_{l}$ is $\delta_{2}$-thin, if $l \geq 4 c_{0}$.

In this case the hypothesis "any continuous injective $\left(1, c_{0}\right)$-quasigeodesic triangle contained in a simple closed geodesic in $S$ is $\delta_{0}$-thin", can be changed by "any geodesic triangle contained in the simple closed geodesic $\gamma$ of $A_{l}$ is $\delta_{0}$-thin", since $T^{\prime \prime}$ is a geodesic triangle in $A_{l}$ if $T$ is a geodesic triangle in $A_{l}$. Since the sides of any geodesic triangle contained in $\gamma$ have length less than or equal to $l / 2$, any geodesic triangle contained in $\gamma$ is $\delta_{0}$-thin, with $\delta_{0}=\delta_{0}\left(A_{l}\right)=l / 4$. Consequently, we obtain that $A_{l}$ is $\delta_{2}$-thin with

$$
\delta_{2}=\max \left\{\frac{l}{4}, 2 \log (1+\sqrt{2})\right\}+K=\frac{l}{4}+K,
$$

since $l / 4 \geq c_{0}>2>2 \log (1+\sqrt{2})$. The same is true for each funnel of $A_{l}$.

Definition 5.3. We say that a non-exceptional Riemann surface $S$ (with or without boundary) is of finite type if its fundamental group is finitely generated. 
Corollary 5.2. Let us consider a non-exceptional Riemann surface $S$ (with or without boundary) of genus 0 ; if $S$ has boundary, we also require that $\partial S$ is the union of local geodesics (closed or non-closed). If $S$ is of finite type, then it is hyperbolic. In fact, if every simple closed geodesic $\gamma$ in $S$ verifies $L(\gamma) \leq l$, then $S$ is $\delta$-thin, with $\delta=\max \left\{\delta\left(4 c_{0}\right), K+(l+\right.$ $\left.\left.c_{0}\right) / 4\right\}$ and $c_{0}, \delta\left(4 c_{0}\right), K$ the constants in Theorem 5.1.

Proof: The set of simple closed geodesics in $S$ is finite: $\left\{\gamma_{1}, \ldots, \gamma_{k}\right\}$, and we have $L\left(\gamma_{j}\right) \leq l$. Every continuous injective $\left(1, c_{0}\right)$-quasigeodesic with its arc-length parametrization $g \subset \gamma_{j}$ verifies $L(g) \leq\left(l+c_{0}\right) / 2$ by Lemma 3.2 ; hence $d(z, \partial g) \leq\left(l+c_{0}\right) / 4$ for every $z \in g$. Then the hypothesis of Theorem 5.1 is verified with $\delta_{0}:=\left(l+c_{0}\right) / 4$. Hence $S$ is $\delta$-thin with $\delta=\max \left\{\delta\left(4 c_{0}\right), K+\left(l+c_{0}\right) / 4\right\}$.

A consequence of this corollary is the following result.

Corollary 5.3. Every generalized $Y$-piece $Y$ with $L\left(\gamma_{i}\right) \leq l$, where $\gamma_{i}$ $(i=1,2,3)$ are the simple closed geodesics in $\partial Y$, is $\delta$-thin, with $\delta=$ $\max \left\{\delta\left(4 c_{0}\right), K+\left(l+c_{0}\right) / 4\right\}$.

Remark. As usual we see a puncture as a simple closed geodesic with zero length.

In order to prove the following result we need one definition.

Definition 5.4. A half-disk is a bordered non-exceptional Riemann surface which is topologically a closed half-plane and whose boundary is a simple geodesic. Every half-disk is conformally equivalent to the subset $\{z \in \mathbf{D}: \Re z \geq 0\}$ of the hyperbolic disk $\mathbf{D}$.

It is clear that a funnel contains infinitely many half-disks.

Two additional consequences which are important in the study of hyperbolicity of Riemann surfaces can be deduced from Theorem 5.1. The first one (see Theorem 5.2 below) allows us to simplify the topology: it assures that to delete funnels and half-disks does not change the hyperbolicity of a Riemann surface. Theorem 5.2 is a useful result which has several applications in [RT2] and [PRT2].

One can think of the following as a natural first result in order to study hyperbolicity: if a Riemann surface has a sequence of funnels $\left\{F_{n}\right\}_{n}$ with $\lim _{n \rightarrow \infty} L\left(\partial F_{n}\right)=\infty$, then it is not hyperbolic. In [RT2] we prove that this reasonable result is false indeed, and an important tool in the proof is Theorem 5.2.

Our recent research let us expect that Theorem 5.2 will be a key tool in the characterization of hyperbolic Denjoy domains. 
Theorem 5.2. Let us consider a non-exceptional Riemann surface $S$ (with or without boundary); if $S$ has boundary, we also require that $\partial S$ is the union of local geodesics (closed or non-closed). Let us denote by $F$ the union of some pairwise disjoint funnels and half-disks of $S$. Let $S_{0}$ be the bordered non-exceptional Riemann surface obtained by deleting from $S$ the interior of $F$. Then $S$ is hyperbolic if and only if $S_{0}$ is hyperbolic.

Furthermore, if $S$ is $\delta$-thin (hyperbolic), then $S_{0}$ is $\delta$-thin (hyperbolic); if $S_{0}$ is $\delta^{\prime}$-hyperbolic, then $S$ is $\delta$-thin, with $\delta=\max \left\{\delta\left(4 c_{0}\right), 4 \delta^{\prime}+\right.$ $\left.2 H\left(\delta^{\prime}, 1, c_{0}\right)+K\right\}, c_{0}, \delta\left(4 c_{0}\right), K$ the constants in Theorem 5.1, and $H$ the constant in Theorem $C$.

Remark. We want to emphasize that there is no hypothesis about the length of the boundary curves of the funnels. This is an important fact since there are hyperbolic Riemann surfaces containing funnels $F_{n}$ with $L\left(\partial F_{n}\right) \longrightarrow \infty$ as $n \longrightarrow \infty$ (see the examples in Section 4 of [RT2]).

Proof: Let us assume that $S$ is $\delta$-thin (hyperbolic). As $S_{0}$ is geodesically convex in $S$ (every geodesic connecting two points of $S_{0}$ is contained in $\left.S_{0}\right)$, then $d_{S}(z, w)=d_{S_{0}}(z, w)$ for every $z, w \in S_{0}$. Therefore $S_{0}$ is also $\delta$-thin (hyperbolic).

Let us assume now that $S_{0}$ is $\delta^{\prime}$-hyperbolic. By Lemma 3.3, every $\left(1, c_{0}\right)$-quasigeodesic triangle $T$ in $S_{0}$ is $\left(4 \delta^{\prime}+2 H\left(\delta^{\prime}, 1, c_{0}\right)\right)$-thin, where $H$ is the constant in Theorem C. Let us observe that any simple closed geodesic in $S$ is contained in $S_{0}$. Since $d_{S}(z, w)=d_{S_{0}}(z, w)$ for every $z, w \in S_{0}$, every $\left(1, c_{0}\right)$-quasigeodesic triangle in $S$ (contained in a simple closed geodesic in $S$ ) is also a $\left(1, c_{0}\right)$-quasigeodesic triangle in $S_{0}$. Let us observe also that $H \geq 1>\log (1+\sqrt{2})$. Then Theorem 5.1 gives that $S$ is $\delta$-thin, with $\delta=\max \left\{\delta\left(4 c_{0}\right), 4 \delta^{\prime}+2 H\left(\delta^{\prime}, 1, c_{0}\right)+K\right\}$.

The following result on geodesically convex subsets of Riemann surfaces is a consequence of the Collar Lemma. It will be useful in the proof of Theorem 5.3.

Lemma 5.5. Let us consider a non-exceptional Riemann surface $S$ (with or without boundary), a simple closed geodesic $\eta$ of $S$ such that $S \backslash \eta$ is not connected, and the closure $S_{0}$ of a connected component of $S \backslash \eta$. We define $L_{0}:=4 \operatorname{Arccosh} t_{0}$, where $t_{0}$ is the unique solution greater than 1 of the equation $2 t^{3}-2 t-1=0$ :

$$
t_{0}:=\sqrt[3]{\frac{9+\sqrt{33}}{36}}+\frac{1}{3} \sqrt[3]{\frac{36}{9+\sqrt{33}}}<1.1915 .
$$

If $L(\eta)<L_{0}$, then every geodesic connecting two points of $S_{0}$ is contained in $S_{0}$, and consequently $d_{S}(z, w)=d_{S_{0}}(z, w)$ for every $z, w \in S_{0}$. 
Proof: We assume first that $S$ is open. If $L:=L(\eta)$, then there exists a collar of $\eta$ of width $d_{0}$ with $\sinh d_{0} \sinh (L / 2)=1$, by the Collar Lemma (see $[\mathbf{R}])$. Hence $\sinh d_{0} \sinh \left(L_{0} / 2\right)>1$, since $L<L_{0}$.

We take $z, w \in S_{0}$. In order to prove the lemma, without loss of generality we can assume that $z, w \in \eta$; therefore $d_{S_{0}}(z, w) \leq L / 2$.

In order to obtain a contradiction, let us assume that there exists a geodesic $\gamma$ in $S$ joining $z, w$, and not contained in $S_{0}$; then $2 d_{0} \leq L(\gamma) \leq$ $L / 2$ and we conclude that $4 d_{0} \leq L$. Let us observe that $2 t^{3}-2 t-1<0$ for every $1<t<t_{0}$; this implies that $2 \cosh ^{3}(L / 4)-2 \cosh (L / 4)<1$, since $L<L_{0}$. Then we have

$$
\begin{aligned}
& 2 \cosh \frac{L_{0}}{4} \sinh ^{2} \frac{L_{0}}{4}<1, \\
& \sinh \frac{L_{0}}{4} \sinh \frac{L_{0}}{2}<1, \\
& \sinh \frac{L_{0}}{4}<\frac{1}{\sinh \frac{L_{0}}{2}}<\sinh d_{0},
\end{aligned}
$$

and hence we obtain $L<4 d_{0}$, which is a contradiction.

If $S$ has boundary, then it is contained in a Riemann surface $R$ and $d_{S}=\left.d_{R}\right|_{S}$. If $\gamma$ is a geodesic in $S$ joining $z, w$, and not contained in $S_{0}$, then there is a geodesic in $R$ joining $z, w$, which is not contained in $S_{0}$, and we have seen that it is a contradiction.

Remark. If we follow the proof of Lemma 5.5, we can deduce that if $L(\eta)=L_{0}$, it is possible for $\gamma$ to escape from $S_{0}$, but then $L(\eta)=2 d_{0}=$ $L / 2$, and we also have $d_{S}(z, w)=d_{S_{0}}(z, w)$ for every $z, w \in S_{0}$.

Many Riemann surfaces can be decomposed in a union of funnels and generalized $Y$-pieces (see [FM, Theorem 4.1] and [AR]). The following result uses this decomposition in order to obtain hyperbolicity. A part of this result appears in [RT1], but here we give a new proof which allows one to obtain an explicit bound for the hyperbolicity constant.

Theorem 5.3. Let us consider a non-exceptional Riemann surface $S$ (with or without boundary) without genus ( $S$ can be viewed as a plane domain). If there is a decomposition of $S$ in a union of funnels $\left\{F_{m}\right\}_{m \in M}$ and generalized $Y$-pieces $\left\{Y_{n}\right\}_{n \in N}$ with $L_{S}(\gamma) \leq l$ for every simple closed geodesic $\gamma \subset\left(\cup_{n} \partial Y_{n}\right) \cup\left(\cup_{m} \partial F_{m}\right)$, then $S$ is $\delta$-hyperbolic, where $\delta:=20 \delta_{0}+l+K_{0}, \delta_{0}:=\max \left\{\delta\left(4 c_{0}\right), K+\left(l+c_{0}\right) / 4\right\}$ and

$$
K_{0}:=\operatorname{Arccosh}\left(\frac{\cosh (l / 2)(1+\cosh (l / 2))}{\sinh ^{2}\left(L_{0} / 2\right)}\right),
$$

with $c_{0}, \delta\left(4 c_{0}\right), K$ the constants in Theorem 5.1 and $L_{0}$ the constant in Lemma 5.5. In fact, if $l<L_{0}$, we can take $\delta:=4 \delta_{0}+l / 2$. 
Proof: First of all, let us observe that $Y_{n}$ is $\delta_{0}$-thin, with $\delta_{0}:=\max \left\{\delta\left(4 c_{0}\right)\right.$, $\left.K+\left(l+c_{0}\right) / 4\right\}$, by Corollary 5.3. Lemma 5.4 and Corollary 5.1 give that $F_{m}$ is also $\delta_{0}$-thin.

We denote by $L_{i}$ for $i=1,2,3$, the three lengths of the simple closed geodesics in $\partial Y_{n}\left(L_{i}=0\right.$ if its corresponding "geodesic" is a puncture).

If $L_{0} \leq L_{i} \leq l$ for at least two geodesics, without loss of generality we can assume that $L_{2}=L_{Y_{n}}\left(\eta_{n m}\right) \geq L_{0}$ and $L_{3}=L_{Y_{n}}\left(\eta_{n k}\right) \geq L_{0}$. We consider the geodesic $g_{m k} \subset Y_{n}$, which joins $\eta_{n m}$ and $\eta_{n k}$, and we put $t=L_{Y_{n}}\left(g_{m k}\right)$. We denote by $\eta_{n r}$ the geodesic in $\partial Y_{n}$ with length $L_{1}$; if we consider the geodesics $g_{m r}, g_{k r}$, joining respectively $\eta_{n m}$ and $\eta_{n r}$, and $\eta_{n k}$ and $\eta_{n r}$, we can split $Y_{n}$ in two isometric right-angle hexagons. By standard hyperbolic trigonometry (see e.g. [B, p. 161], [Ra, p. 100]), we have that

$$
\begin{aligned}
\cosh t & =\frac{\cosh \left(L_{1} / 2\right)+\cosh \left(L_{2} / 2\right) \cosh \left(L_{3} / 2\right)}{\sinh \left(L_{2} / 2\right) \sinh \left(L_{3} / 2\right)} \\
& \leq \frac{\cosh (l / 2)(1+\cosh (l / 2))}{\sinh ^{2}\left(L_{0} / 2\right)}
\end{aligned}
$$

and therefore, $t \leq K_{0}$.

We are going to consider different cases according to the values of $L_{i}$.

(1) If $L_{0} \leq L_{i} \leq l$ for $i=1,2,3$, then the distance between any two simple closed geodesics of $\partial Y_{n}$ is less than or equal to $K_{0}$; therefore $\operatorname{diam}_{Y_{n}}\left(\cup_{m} \eta_{n m}\right) \leq l / 2+K_{0}+l / 2=l+K_{0}$. Then we are in the hypothesis of Theorem 3.1, with $c_{2}=l+K_{0}$ and $B_{n}=\varnothing$.

(2) If $L_{1}<L_{0} \leq L_{2}, L_{3} \leq l$, then the distance between the simple closed geodesics of $\partial Y_{n}$ of length $L_{2}, L_{3}$, (say $\eta_{n m}, \eta_{n k}$ ) is less than or equal to $K_{0}$; then $\operatorname{diam}_{Y_{n}}\left(\eta_{n m} \cup \eta_{n k}\right) \leq l+K_{0}$. Then we are in the hypothesis of Theorem 3.1, with $c_{1}=l / 2, c_{2}=l+K_{0}$ and $A_{n}=\{m, k\}$.

(3) If $L_{1}, L_{2}<L_{0}$, then we are in the hypothesis of Theorem 3.1, with $c_{1}=l / 2$ and $A_{n}=\varnothing$.

The case of $F_{m}$ is similar to (3), with $c_{1}=l / 2$ and $A_{n}=\varnothing$.

Then, Theorem 3.1 (with $c_{1}=l / 2$ and $c_{2}=l+K_{0}$ ) gives that $S$ is $\delta$-thin, with $\delta:=20 \delta_{0}+\max \left\{l / 2+\left(l+K_{0}\right) / 2, l+K_{0}\right\}=20 \delta_{0}+l+K_{0}$.

In fact, if $l<L_{0}$, we only need to consider (3), and then Corollary 3.2 gives that we can take $\delta:=4 \delta_{0}+l / 2$. 


\section{References}

[AR] V. Álvarez And J. M. RodríGuez, Structure theorems for Riemann and topological surfaces, J. London Math. Soc. (2) 69(1) (2004), 153-168.

[ARY] V. Álvarez, J. M. Rodríguez and D. V. Yakubovich, Estimates for nonlinear harmonic "measures" on trees, Michigan Math. J. 49(1) (2001), 47-64.

[An] J. W. Anderson, "Hyperbolic geometry", Springer Undergraduate Mathematics Series, Springer-Verlag London, Ltd., London, 1999.

[BB] Z. M. BAlogh and S. M. Buckley, Geometric characterizations of Gromov hyperbolicity, Invent. Math. 153(2) (2003), 261-301.

[B] A. F. BEARDon, "The geometry of discrete groups", Graduate Texts in Mathematics 91, Springer-Verlag, New York, 1983.

[Bo] M. Bonk, Quasi-geodesic segments and Gromov hyperbolic spaces, Geom. Dedicata 62(3) (1996), 281-298.

[BHK] M. Bonk, J. Heinonen and P. Koskela, Uniformizing Gromov hyperbolic spaces, Astérisque 270 (2001), 99 pp.

[Bu] P. BUSER, "Geometry and spectra of compact Riemann surfaces", Progress in Mathematics 106, Birkhäuser Boston, Inc., Boston, MA, 1992.

[CFPR] A. Cantón, J. L. Fernández, D. Pestana and J. M. RoDRÍGuez, On harmonic functions on trees, Potential Anal. 15(3) (2001), 199-244.

[C] I. Chavel, "Eigenvalues in Riemannian geometry", Including a chapter by Burton Randol, With an appendix by Jozef Dodziuk, Pure and Applied Mathematics 115, Academic Press, Inc., Orlando, FL, 1984.

[CDP] M. Coornaert, T. Delzant and A. Papadopoulos, "Géométrie et théorie des groupes. Les groupes hyperboliques de Gromov", Lecture Notes in Mathematics 1441, Springer-Verlag, Berlin, 1990.

[F] W. Fenchel, "Elementary geometry in hyperbolic space", With an editorial by Heinz Bauer, de Gruyter Studies in Mathematics 11, Walter de Gruyter \& Co., Berlin, 1989.

[FM] J. L. Fernández And M. V. Melián, Escaping geodesics of Riemannian surfaces, Acta Math. 187(2) (2001), 213-236. 
[FR1] J. L. Fernández and J. M. Rodríguez, The exponent of convergence of Riemann surfaces. Bass Riemann surfaces, Ann. Acad. Sci. Fenn. Ser. A I Math. 15(1) (1990), 165-183.

[FR2] J. L. Fernández And J. M. Rodríguez, Area growth and Green's function of Riemann surfaces, Ark. Mat. 30(1) (1992), 83-92.

[GH] É. Ghys and P. DE la HaRpe, eds., "Sur les groupes hyperboliques d'après Mikhael Gromov", Papers from the Swiss Seminar on Hyperbolic Groups held in Bern, 1988, Progress in Mathematics 83, Birkhäuser Boston, Inc., Boston, MA, 1990.

[G1] M. Gromov, Hyperbolic groups, in: "Essays in group theory”, Math. Sci. Res. Inst. Publ. 8, Springer, New York, 1987, pp. $75-263$.

[G2] M. Gromov, "Metric structures for Riemannian and nonRiemannian spaces", Based on the 1981 French original, With appendices by M. Katz, P. Pansu and S. Semmes, Translated from the French by Sean Michael Bates, Progress in Mathematics 152, Birkhäuser Boston, Inc., Boston, MA, 1999.

[HS] I. Holopainen ANd P. M. SoARd, $p$-harmonic functions on graphs and manifolds, Manuscripta Math. 94(1) (1997), 95-110.

[JS] G. A. Jones and D. Singerman, "Complex functions. An algebraic and geometric viewpoint", Cambridge University Press, Cambridge, 1987.

[K1] M. KANAI, Rough isometries, and combinatorial approximations of geometries of noncompact Riemannian manifolds, $J$. Math. Soc. Japan 37(3) (1985), 391-413.

[K2] M. KANAI, Rough isometries and the parabolicity of Riemannian manifolds, J. Math. Soc. Japan 38(2) (1986), 227-238.

[K3] M. KANAI, Analytic inequalities, and rough isometries between noncompact Riemannian manifolds, in: "Curvature and topology of Riemannian manifolds" (Katata, 1985), Lecture Notes in Math. 1201, Springer, Berlin, 1986, pp. 122-137.

[N] P. J. Nicholls, "The ergodic theory of discrete groups", London Mathematical Society Lecture Note Series 143, Cambridge University Press, Cambridge, 1989.

[P] F. PAulin, On the critical exponent of a discrete group of hyperbolic isometries, Differential Geom. Appl. 7(3) (1997), 231-236. 
[PRT1] A. Portilla, J. M. Rodríguez and E. Tourís, Gromov hyperbolicity through decomposition of metrics spaces. II, $J$. Geom. Anal. 14(1) (2004), 123-149.

[PRT2] A. Portilla, J. M. Rodríguez and E. Tourís, The topology of balls and Gromov hyperbolicity of Riemann surfaces, Differential Geom. Appl. 21(3) (2004), 317-335.

[R] B. Randol, Cylinders in Riemann surfaces, Comment. Math. Helv. 54(1) (1979), 1-5.

[Ra] J. G. RATCliffe, "Foundations of hyperbolic manifolds", Graduate Texts in Mathematics 149, Springer-Verlag, New York, 1994.

[R1] J. M. RodríGuez, Isoperimetric inequalities and Dirichlet functions of Riemann surfaces, Publ. Mat. 38(1) (1994), $243-253$

[R2] J. M. Rodríguez, Two remarks on Riemann surfaces, Publ. Mat. 38(2) (1994), 463-477.

[RT1] J. M. RodríGuez And E. Tourís, Gromov hyperbolicity through decomposition of metric spaces, Acta Math. Hungar. 103(1-2) (2004), 107-138.

[RT2] J. M. RodríGuEZ AND E. Tourís, Gromov hyperbolicity of Riemann surfaces, Acta Math. Sin. (Engl. Ser.) (to appear).

[S] H. Shimizu, On discontinuous groups operating on the product of the upper half planes, Ann. of Math. (2) 77 (1963), 33-71.

[So] P. M. SOARDI, Rough isometries and Dirichlet finite harmonic functions on graphs, Proc. Amer. Math. Soc. 119(4) (1993), 1239-1248.

Departamento de Matemáticas

Universidad Carlos III de Madrid

Avenida de la Universidad, 30

28911 Leganés, Madrid

Spain

E-mail address: jomaro@math.uc3m.es

E-mail address: etouris@math.uc3m.es

Primera versió rebuda el 18 de gener de 2005, darrera versió rebuda el 23 de febrer de 2006. 